\title{
Numerical Approximation to Nonlinear One Dimensional Coupled Reaction Diffusion System
}

\author{
Shahid Hasnain, Daoud Suleiman Mashat, Muhammad Saqib, Shafeek A. Ghaleb, \\ Noorah Y. Mshary \\ Department of Mathematics, Numerical Analysis, King Abdulaziz University, Jeddah, Saudi Arabia \\ Email: shahidqa32@gmail.com,dmashat@kau.edu.sa,saqibmurree@gmail.com,Shafeekye2@gmailcom, \\ noorahmshary1@gmail.com
}

How to cite this paper: Hasnain, S., Mashat, D.S., Saqib, M., Ghaleb, S.A. and Mshary, N.Y. (2017) Numerical Approximation to Nonlinear One Dimensional Coupled Reaction Diffusion System. Journal of Applied Mathematics and Physics, $\mathbf{5}$, 1551-1574.

https://doi.org/10.4236/jamp.2017.58129

Received: April 8, 2017

Accepted: August 22, 2017

Published: August 28, 2017

Copyright () 2017 by authors and Scientific Research Publishing Inc. This work is licensed under the Creative Commons Attribution International License (CC BY 4.0).

http://creativecommons.org/licenses/by/4.0/

\begin{abstract}
This research paper represents a numerical approximation to non-linear coupled one dimension reaction diffusion system, which includes the existence and uniqueness of the time dependent solution with upper and lower bounds of the solution. Also numerical approximation is obtained by finite difference schemes to reach at reasonable level of accuracy, which is magnified by $L_{2}$, $L_{\infty}$ and relative error norms. The accuracy of the approximations is shown by randomly selected grid points along time level and comparison with analytical results. The test example demonstrates the accuracy, efficiency and versatility of the proposed schemes. Moreover, the schemes can be easily applied to a wide class of higher dimension non-linear reaction diffusion equations with a little modifications.
\end{abstract}

\section{Keywords}

Forward in Time and Centre in Space (FTCS), Taylor's Series, Crank Nicolson, Fourth Order Implicit Scheme and Richardson Extrapolation

\section{Introduction}

Fisher and Kolmogorov Petrovsky Piscounov founded quasilinear partial differential equation which represents reaction diffusion phenomena [1] [2], which play important rule in population dynamics as propagation of beneficial genes; mathematical description to this phenomena is as follows [1] [2] [3],

$$
\partial_{t} u=-\partial_{x} i+Q(\rho) \text {, }
$$


here, $x$ declares spatial coordinate position with time $t$, also $\rho(x, t)$ is the term used to identify the population density, $i$ is the flux associated to population, and $Q(\rho)$ is a limited to originator term that illuminate the net rate of production or growth in the populated area or density associated to population [3]. The behaviour of the diffusion is accounted in the flux $i$, inclines by Fick's law,

$$
i=-D \partial_{x} \rho,
$$

where the assumption on the diffusion coefficient $D$, is to be constant [3]. It is frequently to imitate the law, which is known as the Pearl-Verhulst logistic law,

$$
Q(\rho) \Rightarrow \begin{cases}\gamma \rho\left(1-\frac{\rho}{\rho_{s}}\right), & 0<\rho<\rho_{s} \\ 0, & \rho_{s} \leq \rho \leq 0\end{cases}
$$

related to stranded scale to growth in the population [3]. This law explains the that initially population would grow with homogeneous fashion with time of $\gamma^{-1}$ until that growth reach at some stranded level with some saturation in population [3]. Fisher and KPP were very much keen about the speed that exists in advantageous genes to propagate and they found a travelling wave solution to Equations (1), (2), (3) [3], such solution related to velocity $\geq 2(D \gamma)^{1 / 2}$. Consequential, Kametaka (1976) and Uchiyama (1977)] remarks about velocity of $2(D \gamma)^{1 / 2}$ developed initial conditions (ICs), categorised as [3],

$$
\left.\begin{array}{l}
\rho(x, t=0)=\rho_{s}, \text { for } x \leq x_{1} \\
0<\rho(x, t=0)<\rho_{s}, \text { for } x_{1}<x<x_{2} \\
\rho(x, t=0)=0, \text { for } x_{2} \leq x
\end{array}\right\}
$$

\section{Governing Equation}

In the year of $1948 \mathrm{El}$ ' dovich, raised the major features of the diffusion reaction semi-linear equation which make useful contribution to wave of advantageous genes which are now totally classify as in the dynamics of the gas and flame with chemical kinetics [3]. Mostly, in process of combustion, the concept of heat which is channelized by the conduction processed by heat related to molecules which moves from the hot part of flame to coolant side [3], by a particular locality, the rate of the chemical reaction such as exothermic chemical reaction (due to presence of heat), totally aware of temperature of the related gas mixture [3]. The coefficients associated with molecular diffusion and thermal are at same level of scaled, the temperature and concentration are situated almost isobaric [3]. The quantity $\rho$, then can represent either the temperature or concentration, without confusion, and is often referred to as the "combustion completeness by such coefficient" by Vulis, 1961 [3]. Equations (1)-(4) are then hired to modelled this attractive phenomena as a result of travelling wave nature of the flame exists in combustion process or zone, i.e. the transition layer between the region of burnt gas and the gas where combustion has not yet begun [3]. Above literature review, leads to the following reaction-diffusion system in two components 
along single direction, is as follows,

$$
\left.\begin{array}{l}
\partial_{t} u=\boldsymbol{\beta}_{1} u_{x x}+\boldsymbol{\alpha}_{1} u(1-u)(1-v) \\
\partial_{t} v=\boldsymbol{\beta}_{2} v_{x x}+\boldsymbol{\alpha}_{2} v(1-v)(1-u)
\end{array}\right\}
$$

where in above Equation (5), $\boldsymbol{\alpha}_{1}$ and $\boldsymbol{\alpha}_{2}$ are the linear growth rate whereas $\boldsymbol{\beta}_{1}, \boldsymbol{\beta}_{2}$ are coefficients of the diffusion [3] [4] [5], with $\boldsymbol{\alpha}_{1}, \boldsymbol{\alpha}_{2}, \boldsymbol{\beta}_{1}, \boldsymbol{\beta}_{2}>0$, as well as $u(x, t)$ and $v(x, t)$ are the concentrations of the chemicals in the reaction diffusion process.

\section{Exact Solution}

Exact solution to system in Equation (5) found by E. S. Fahmy [5], he used factorization method, given below:

$$
\left.\begin{array}{l}
u^{+}(z)=\frac{1}{2}+\frac{1}{2} \tanh \left(D_{1}\right) \\
u^{-}(z)=\frac{1}{2}-\frac{1}{2} \operatorname{coth}\left(D_{1}\right)
\end{array}\right\} \text { where } D_{1}=\frac{k_{1}}{2}\left(z-z_{0}\right)
$$

\section{Smoothness and Uniqueness of the Reaction Diffusion System}

It is very important to enforce some assumptions on some physical parameters or related reaction functions, to analysed the smoothness and uniqueness of a positive oriented natural solution by considering lower and upper bound of the solution of the system which mentioned in Equation (5) [6]. We assume that $\boldsymbol{\beta}_{1}=\boldsymbol{\beta}_{2}$ which represents the diffusion coefficients are always positive in the bounded or finite domain such as $\Omega$, whereas initially means at $t=0$ the unknowns such as $\left(u_{0}, v_{0}\right)$ are non-negative [6]. The smoothness hypothesis is used to guarantee the existence of the problem of the corresponding linear system [6]. Let us look at the complete obtained system in one dimension,

$$
\left.\begin{array}{l}
u_{t}-\nabla \cdot\left(\boldsymbol{\beta}_{1} \nabla u\right)=\boldsymbol{\alpha}_{1} u^{m} g(x, u, v) \\
v_{t}-\nabla \cdot\left(\boldsymbol{\beta}_{2} \nabla v\right)=\boldsymbol{\alpha}_{2} v^{m} g(x, u, v)
\end{array}\right\} \text { for } m \geq 1, g(x, u, v)=(1-u)(1-v) .
$$

imposed the following boundary conditions on Equation (8), which is,

$$
\left.\begin{array}{l}
B_{1}[u]=h_{1}(x) \\
B_{2}[v]=h_{2}(x)
\end{array}\right\} \text { for } t>0,(x) \in \Omega
$$

imposed the following initial conditions on Equation (8), which is,

$$
\left.\begin{array}{l}
u(0, x)=u_{0}(x) \\
v(0, x)=v_{0}(x)
\end{array}\right\} \text { for } x \in \Omega
$$

combined above three equations, to get the model system. 


\section{Assumption or Hypothesis ( $\mathrm{H})$}

Let us suppose that $f_{v}$ exists and is also bounded which is subsets of domain $\Omega$ and there exists a function with $C_{o}(x) \geq 0$, such that

$0 \leq f\left(u, v_{1}\right) \leq f\left(u, v_{2}\right) \leq c_{o}(x)$ for $0 \leq v_{1} \leq v_{2} \leq \infty$ [6]. This definition implies that the function $\mathrm{f}$ is monotone non-decreasing in $v$ and is uniform bounded for $v \geq 0$ [6]. Clearly this condition is satisfied by function $\mathrm{f}$ [6]. Thus this property leads to

$$
\begin{gathered}
G_{1}(u, v)=\alpha_{1} u^{m} g(x, u, v) \\
G_{2}(u, v)=\boldsymbol{\alpha}_{1} v^{m} g(x, u, v),
\end{gathered}
$$

where above Equations (11), (12) represents $G_{1}, G_{2}$ are quasi monotone nonincreasing and quasi monotone non-decreasing functions in $\Omega$ respectively [6]. According to classification of the reaction functions, $G_{1}, G_{2}$ are typed III functions [6]. This leads to the following definition of the solutions.

\subsection{Definition}

A smooth pair of two vector functions $(\breve{u}, \breve{v}),(\bar{u}, \bar{v})$ defined in $\mathbf{R}^{+} \times \Omega$ are called upper and lower solutions respectively [6], if they satisfy the following inequalities

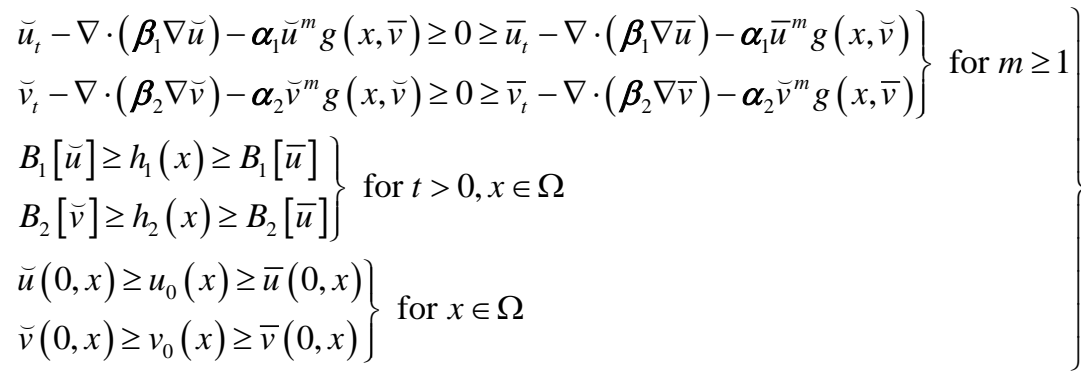

In the above definitions the smoothness of $(\breve{u}, \breve{v}),(\bar{u}, \bar{v})$ is in the sense that these functions are continuously differentiable to the order appeared in Equations (8) and (11), (12) respectively [6]. Hypothesis and above definition leads to the following theorem.

\subsection{Theorem}

Let $\mathrm{f}$ holds hypothesis $(\mathrm{H})$. If there exist upper and lower solutions $(\breve{u}, \breve{v})$, $(\bar{u}, \bar{v})$ of system (8), with $\breve{u} \leq \bar{u}$ and $\breve{v} \leq \bar{v}$ in $\mathbf{R}^{+} \times \Omega$, then the sequence $\left\{\dot{u}^{k}, \dot{v}^{k}\right\},\left\{\underline{u}^{k}, \underline{v}^{k}\right\}$ converges monotonically from above and below, respectively [6], to a unique solution (u, v) of system (8) [6]. Moreover,

$$
\left.\begin{array}{l}
\breve{u}(t, x) \leq u(t, x) \leq \bar{u}(t, x) \\
\breve{v}(t, x) \leq v(t, x) \leq \bar{v}(t, x)
\end{array}\right\} t>0, x \in \Omega,
$$

The quality of being of practical use about above theorem, need to construct of lower and upper solutions, with existence problem to be ensured [6], but the asymptotic behaviour of the time-dependent solution and related stability can 
also be established from the behaviour of the lower and upper solutions [6], according to the definition, so stability of a steady-state solution make according to selection of Lyapunov function [6], be that as it may for the global existence problem or the stability problem with homogeneous boundary conditions [6].

\section{Numerical Methods}

Let us apply numerical methods technique, to solve such system which mentioned in Equation (5) in finite domain $\Omega$. We partitioned the interval $[a, b]$ into $\mathrm{n}$ equal parts of width $\mathrm{h}$ [7] [8]. Place a grid on the rectangle region $\mathrm{R}$ by drawing vertical and horizontal lines through the points. Numerical solution is denoted by $\left(u_{m}^{n}, v_{m}^{n}\right)$, whereas exact one make in the form of $\left(U_{m}^{n}, V_{m}^{n}\right)$ respectively.

\subsection{Forward in Time and Centre in Space (FTCS) Scheme}

We consider forward in time and center in space (FTCS) explicit scheme by substituting the forward difference approximation for the time derivative and the central difference approximation for the space derivative in system in Equation (5),

$$
\left.\begin{array}{l}
\begin{array}{l}
u_{i}^{n+1}-u_{i}^{n} \\
k
\end{array}=\frac{\beta_{1}}{h^{2}}\left(u_{i+1}^{n}-2 u_{i}^{n}+u_{i-1}^{n}\right)+\alpha_{1} u_{i}^{n}\left(1-u_{i}^{n}\right)\left(1-v_{i}^{n}\right) \\
\frac{v_{i}^{n+1}-v_{i}^{n}}{k}=\frac{\beta_{2}}{h^{2}}\left(v_{i+1}^{n}-2 v_{i}^{n}+v_{i-1}^{n}\right)+\alpha_{2} v_{i}^{n}\left(1-v_{i}^{n}\right)\left(1-u_{i}^{n}\right)
\end{array}\right\}
$$

where $\mathbf{R}_{1}=\frac{k \beta_{1}}{h^{2}}, \mathbf{R}_{2}=\frac{k \beta_{2}}{h^{2}}, \mathbf{Q}_{1}=k \alpha_{1}$ and $\mathbf{Q}_{2}=k \alpha_{2}$.

Finite difference schemes, must pass certain tests of accuracy, consistency, stability and convergence [9] [10] [11], we discuss these concepts in the following way.

\subsubsection{Accuracy of FTCS}

Accuracy of the FTCS scheme for system in Equation (16), we apply Taylor's series on each term, which is as follows:

$$
\left.\begin{array}{l}
u_{\text {Resultant }}=\left(u_{t}-u_{x x}-u(1-u)(1-v)\right) k+\frac{1}{2} k^{2} u_{t t}-\frac{1}{12} k h^{2} u_{x x x x}+\frac{1}{6} k^{3} u_{t t t}+\cdots \\
v_{\text {Resultant }}=\left(v_{t}-v_{x x}-v(1-v)(1-u)\right) k+\frac{1}{2} k^{2} v_{t t}-\frac{1}{12} k h^{2} v_{x x x x}+\frac{1}{6} k^{3} v_{t t t}+\cdots
\end{array}\right\}
$$

simplifies above equations, we get the following,

$$
\left.\begin{array}{l}
u_{\text {Resultant }}=\frac{1}{2} k^{2} u_{t t}-\frac{1}{12} k h^{2} u_{x x x x}+\frac{1}{6} k^{3} u_{t t t}+\cdots \\
v_{\text {Resultant }}=\frac{1}{2} k^{2} v_{t t}-\frac{1}{12} k h^{2} v_{x x x x}+\frac{1}{6} k^{3} v_{t t t}+\cdots
\end{array}\right\}
$$

dividing above system, by $\mathrm{k}$ and simplifies some terms: 


$$
\left.\begin{array}{l}
u_{\text {Resultant }}=\frac{1}{2} k u_{t t}-\frac{1}{12} h^{2} u_{x x x x}+\frac{1}{6} k^{2} u_{t t t}+\cdots \\
v_{\text {Resultant }}=\frac{1}{2} k v_{t t}-\frac{1}{12} h^{2} v_{x x x x}+\frac{1}{6} k^{2} v_{t t t}+\cdots
\end{array}\right\}
$$

This leads to principle part of the truncation error (PPTE), which is as follows:

$$
\left.\begin{array}{l}
\operatorname{PPTE}_{u}=\frac{1}{2} k u_{t t}-\frac{1}{12} h^{2} u_{x x x x}+\frac{1}{6} k^{2} u_{t t t}+\cdots \\
\operatorname{PPTE}_{v}=\frac{1}{2} k v_{t t}-\frac{1}{12} h^{2} v_{x x x x}+\frac{1}{6} k^{2} v_{t t t}+\cdots
\end{array}\right\}
$$

which shows that this scheme has 2 nd order accuracy in space and first order accuracy in time such as $O\left(k, h^{2}\right)$ [9].

\subsubsection{Consistency of FTCS}

According to the definition of the consistency, if the difference between finite difference equation (FDE) and related partial differential equation (PDE), i.e truncation error vanishes as the sizes of the grid spacing go to zero independently, i.e

$$
\frac{\text { Truncation error }}{k} \rightarrow 0 \text { as } h, k \rightarrow 0
$$

Equation (20) is consistent, because the truncation error divided by $k$ tends to zero as $\mathrm{h}$ and $\mathrm{k}$ tends to zero [11] [12] [13] [14].

\subsubsection{Convergence of FTCS}

A finite difference method is convergent if the solution of the finite difference equation approaches to exact solution of the partial differential equation as the sizes of the grid spacing $h$ and $k$ tends to zero [13] [14] [15].

$$
u_{m}^{n}-U_{m}^{n}=z_{m}^{n} \rightarrow 0 \text { as } h, k \rightarrow 0
$$

where $z_{m}^{n}$ is called the discretization error.

$$
v_{m}^{n}-V_{m}^{n}=Z_{m}^{n} \rightarrow 0 \text { as } h, k \rightarrow 0
$$

where $Z_{m}^{n}$ is called the discretization error.

$$
\begin{aligned}
\left|u_{m}^{n}-U_{m}^{n}\right| & \rightarrow 0, \text { as } h, k \rightarrow 0 \\
\left|v_{m}^{n}-V_{m}^{n}\right| & \rightarrow 0 \text {, as } h, k \rightarrow 0
\end{aligned}
$$

this completes the proof of the convergence.

\subsubsection{Stability of FTCS}

Another important feature of a finite difference method of solving partial differential equation is the stability of the associated finite difference equation which must be investigated. Let us look again Equation (16),

$$
\left.\begin{array}{l}
u_{i}^{n+1}=u_{i}^{n}+\mathbf{R}_{1}\left(u_{i+1}^{n}-2 u_{i}^{n}+u_{i-1}^{n}\right)+\mathbf{Q}_{1} u_{i}^{n}\left(1-u_{i}^{n}\right)\left(1-v_{i}^{n}\right) \\
\left.v_{i}^{n+1}=v_{i}^{n}+\mathbf{R}_{2}\left(v_{i+1}^{n}-2 v_{i}^{n}+v_{i-1}^{n}\right)+\mathbf{Q}_{2} v_{i}^{n}\left(1-v_{i}^{n}\right)\left(1-u_{i}^{n}\right)\right)
\end{array}\right\}
$$


linear form of above Equation (26), is as follows:

$$
\left.\begin{array}{c}
u_{i}^{n+1}=u_{i}^{n}+\mathbf{R}_{1}\left(\delta_{x}^{2} u_{m}^{n}\right)+\mathbf{Q}_{1} u_{m}^{n} \mathbf{A} \\
v_{i}^{n+1}=v_{i}^{n}+\mathbf{R}_{2}\left(\delta_{x}^{2} v_{m}^{n}\right)+\mathbf{Q}_{2} v_{m}^{n} \mathbf{A}
\end{array}\right\}
$$

where $\mathbf{A}=\max \left[\left(1-u_{m}^{n}\right)\left(1-v_{m}^{n}\right)\right]$, from Equations (26) and (27), we can write in the following way,

$$
\left[\begin{array}{ll}
1 & 0 \\
0 & 1
\end{array}\right]\left[\begin{array}{l}
u^{n+1} \\
v^{n+1}
\end{array}\right]=\left[\begin{array}{cc}
C_{1} & 0 \\
0 & C_{1}
\end{array}\right]\left[\begin{array}{l}
u^{n} \\
v^{n}
\end{array}\right]
$$

where $C_{1}=1-4 \mathbf{R}_{1} \sin ^{2}(\beta h / 2)+\mathbf{A} \mathbf{Q}_{1}$.

$$
\psi^{n+1}=G \psi^{n}
$$

where

$$
G=\left[\begin{array}{ll}
1 & 0 \\
0 & 1
\end{array}\right]^{-1}\left[\begin{array}{cc}
C_{1} & 0 \\
0 & C_{1}
\end{array}\right]
$$

where matrix $G$ is called the amplification matrix. According to Von-Neumann stability analysis, the condition for stability for system is

$$
\max \mid\{\text { eigenvalues of } G\} \mid \leq 1
$$

above Inequality (29) leads to the following two special cases,

1) Special Case 1

- The right hand side of the Inequity (29) gives,

$$
\left.\begin{array}{l}
1-4 \mathbf{R}_{1} \sin ^{2}\left(\frac{\beta h}{2}\right)+\mathbf{A} \mathbf{Q}_{1} \leq 1 \\
0-4 \mathbf{R}_{1} \sin ^{2}\left(\frac{\beta h}{2}\right)+\mathbf{A} \mathbf{Q}_{1} \leq 0 \\
4 \mathbf{R}_{1} \sin ^{2}\left(\frac{\beta h}{2}\right) \geq \mathbf{A} \mathbf{Q}_{1} \\
\mathbf{R}_{1} \geq \frac{\mathbf{A} \mathbf{Q}_{1}}{4 \sin ^{2}\left(\frac{\beta h}{2}\right)}
\end{array}\right\}
$$

which is condition of stability to FTCS scheme in coupled non-linear PDE system.

2) Special Case 2

- The left hand side of the Inequity (29) gives,

$$
\left.\begin{array}{l}
-1 \leq 1-4 \mathbf{R}_{1} \sin ^{2}\left(\frac{\beta h}{2}\right)+\mathbf{A} \mathbf{Q}_{1} \\
-2 \leq-4 \mathbf{R}_{1} \sin ^{2}\left(\frac{\beta h}{2}\right)+\mathbf{A} \mathbf{Q}_{1} \\
4 \mathbf{R}_{1} \sin ^{2}\left(\frac{\beta h}{2}\right) \leq 2+\mathbf{A} \mathbf{Q}_{1} \\
\mathbf{R}_{1} \leq \frac{2+\mathbf{A} \mathbf{Q}_{1}}{4 \sin ^{2}\left(\frac{\beta h}{2}\right)}
\end{array}\right\}
$$


which is condition of stability to FTCS scheme in coupled non-linear PDE system.

Special case (1) and special case (2) lead to the following very interesting results,

$$
\frac{\mathbf{A} \mathbf{Q}_{1}}{4 \sin ^{2}\left(\frac{\beta h}{2}\right)} \leq \mathbf{R}_{1} \leq \frac{2+\mathbf{A} \mathbf{Q}_{1}}{4 \sin ^{2}\left(\frac{\beta h}{2}\right)}
$$

The Von-Neumann stability analysis is the most common used method of determining stability criterion as it is generally the easiest to apply. It can only be used to establish a necessary and sufficient condition for stability of linear initial value problems with constant coefficients [14] [15] [16] [17] [18] [19]. Thus according to Von-Neumann stability analysis, FTCS scheme is conditionally stable as obtained in Equation (32).

\subsection{Crank Nicolson Implicit Scheme}

Let us apply Crank Nicolson implicit finite difference scheme to Equation (5).

$$
\left.\begin{array}{rl}
\frac{u_{i}^{n+1}-u_{i}^{n}}{k}+O\left(k^{2}\right)= & \frac{1}{2 h^{2}}\left[\delta_{x}^{2} u_{i}^{n+1}+\delta_{x}^{2} u_{i}^{n}+O\left(k^{2}+O\left(h^{2}\right)\right]\right. \\
& +\frac{u_{i}^{n+1}+u_{i}^{n}}{2}\left(1-\frac{u_{i}^{n+1}+u_{i}^{n}}{2}\right)\left(1-\frac{v_{i}^{n+1}+v_{i}^{n}}{2}\right) \\
\frac{v_{i}^{n+1}-v_{i}^{n}}{k}+O\left(k^{2}\right)= & \frac{1}{2 h^{2}}\left[\delta_{x}^{2} v_{i}^{n+1}+\delta_{x}^{2} v_{i}^{n}+O\left(k^{2}+O\left(h^{2}\right)\right]\right. \\
& +\frac{v_{i}^{n+1}+v_{i}^{n}}{2}\left(1-\frac{v_{i}^{n+1}+v_{i}^{n}}{2}\right)\left(1-\frac{u_{i}^{n+1}+u_{i}^{n}}{2}\right)
\end{array}\right\}
$$

after some simplification, we get the following,

$$
\begin{aligned}
& \left(1-\frac{1}{2} \mathbf{R}_{1} \delta_{x}^{2}\right) u_{i}^{n+1}=\left(1+\frac{1}{2} \mathbf{R}_{1} \delta_{x}^{2}\right) u_{i}^{n}+\frac{1}{2} \mathbf{Q}_{1}\left(u_{i}^{n+1}+u_{i}^{n}\right)\left(1-\left(\frac{u_{i}^{n+1}+u_{i}^{n}}{2}\right)\right)\left(1-\left(\frac{v_{i}^{n+1}+v_{i}^{n}}{2}\right)\right) \\
& \left(1-\frac{1}{2} \mathbf{R}_{2} \delta_{x}^{2}\right) v_{i}^{n+1}=\left(1+\frac{1}{2} \mathbf{R}_{2} \delta_{x}^{2}\right) v_{i}^{n}+\frac{1}{2} \mathbf{Q}_{2}\left(v_{i}^{n+1}+v_{i}^{n}\right)\left(1-\left(\frac{v_{i}^{n+1}+v_{i}^{n}}{2}\right)\right)\left(1-\left(\frac{u_{i}^{n+1}+u_{i}^{n}}{2}\right)\right)
\end{aligned}
$$

combine Equations (34) (35), we get the following,

$$
\left.\begin{array}{l}
\left(1-\frac{1}{2} \mathbf{R}_{1} \delta_{x}^{2}\right) u_{i}^{n+1}=\left(1+\frac{1}{2} \mathbf{R}_{1} \delta_{x}^{2}\right) u_{i}^{n}+\frac{1}{2} \mathbf{Q}_{1}\left(u_{i}^{n+1}+u_{i}^{n}\right)\left(1-\left(\frac{u_{i}^{n+1}+u_{i}^{n}}{2}\right)\right)\left(1-\left(\frac{v_{i}^{n+1}+v_{i}^{n}}{2}\right)\right) \\
\left(1-\frac{1}{2} \mathbf{R}_{2} \delta_{x}^{2}\right) v_{i}^{n+1}=\left(1+\frac{1}{2} \mathbf{R}_{2} \delta_{x}^{2}\right) v_{i}^{n}+\frac{1}{2} \mathbf{Q}_{2}\left(v_{i}^{n+1}+v_{i}^{n}\right)\left(1-\left(\frac{v_{i}^{n+1}+v_{i}^{n}}{2}\right)\right)\left(1-\left(\frac{u_{i}^{n+1}+u_{i}^{n}}{2}\right)\right)
\end{array}\right\}
$$

where $\mathbf{R}_{1}, \mathbf{R}_{2}, \mathbf{Q}_{1}$ and $\mathbf{Q}_{2}$ are mentioned in Equation (16).

\subsubsection{Accuracy of CN}

Accuracy of the CN scheme to Equation (36), we apply Taylor's series on each term. After some simplification, resultant is as follows, 


$$
\begin{aligned}
u_{\text {Resultant }}= & \left(u_{t}-u_{x x}-u(1-u)(1-v)\right) k+\frac{1}{2} k^{2}\left[u_{t t}-u_{x x t}+u v_{t}(1-u)-u_{t}(1-u)(1-v)\right. \\
& \left.+u u_{t}(1-v)\right]-\frac{1}{12} k h^{2} u_{x x x x}+k^{3}\left(\frac{1}{6} u_{t t t}-\frac{1}{4} u_{x x t t}+\cdots\right)-\frac{1}{24} k^{2} h^{2} u_{x x x x t}+\cdots \\
v_{\text {Resultant }}= & \left(v_{t}-v_{x x}-v(1-v)(1-u)\right) k+\frac{1}{2} k^{2}\left[v_{t t}-v_{x x t}+v u_{t}(1-v)-v_{t}(1-v)(1-u)\right. \\
& \left.+v v_{t}(1-u)\right]-\frac{1}{12} k h^{2} v_{x x x x}+k^{3}\left(\frac{1}{6} v_{t t t}-\frac{1}{4} v_{x x t t}+\cdots\right)-\frac{1}{24} k^{2} h^{2} v_{x x x x t}+\cdots
\end{aligned}
$$

we divide above equation by time step $k$ with using in system (5), to get the accuracy, in the following form,

$$
\left.\begin{array}{c}
u_{\text {Resultant }}=\frac{1}{2} k \frac{\partial}{\partial t}\left[u_{t}-u_{x x}-u(1-u)(1-v)\right]-\frac{1}{12} h^{2} u_{x x x x} \\
+k^{2}\left(\frac{1}{6} u_{t t t}-\frac{1}{4} u_{x x t t}+\cdots\right)-\frac{1}{24} k h^{2} u_{x x x x t}+\cdots \\
v_{\text {Resultant }}=\frac{1}{2} k \frac{\partial}{\partial t}\left[v_{t}-v_{x x}-v(1-v)(1-u)\right]-\frac{1}{12} h^{2} v_{x x x x} \\
+k^{2}\left(\frac{1}{6} v_{t t t}-\frac{1}{4} v_{x x t t}+\cdots\right)-\frac{1}{24} k h^{2} v_{x x x x t}+\cdots \\
u_{\text {Resultant }}=-\frac{1}{12} h^{2} u_{x x x x}+k^{2}\left(\frac{1}{6} u_{t t t}-\frac{1}{4} u_{x x t t}+\cdots\right)-\frac{1}{24} k h^{2} u_{x x x x t}+\cdots \\
v_{\text {Resultant }}=-\frac{1}{12} h^{2} v_{x x x x}+k^{2}\left(\frac{1}{6} v_{t t t}-\frac{1}{4} v_{x x t t}+\cdots\right)-\frac{1}{24} k h^{2} v_{x x x x t}+\cdots
\end{array}\right\}
$$

Now principle part of the truncation error $(P P T E)$ is as follows:

$$
\left.\begin{array}{l}
P P T E_{u}=-\frac{1}{12} h^{2} u_{x x x x}+k^{2}\left(\frac{1}{6} u_{t t t}-\frac{1}{4} u_{x x t t}+\cdots\right)-\frac{1}{24} k h^{2} u_{x x x x t}+\cdots \\
P P T E_{v}=-\frac{1}{12} h^{2} v_{x x x x}+k^{2}\left(\frac{1}{6} v_{t t t}-\frac{1}{4} v_{x x t t}+\cdots\right)-\frac{1}{24} k h^{2} v_{x x x x t}+\cdots
\end{array}\right\}
$$

which shows that this scheme is 2 nd order accurate in both time and space, such as $O\left(k^{2}, h^{2}\right)$ [19] [20].

\subsubsection{Consistency of $\mathrm{CN}$}

From accuracy, we find principle part of the truncation error along with Equation (42). Which shows that Crank Nicolson scheme is consistent because $z_{m}^{n}=P P T E$ tends to zero as $h$ and $k$ tends to zero, i.e. $z_{m}^{n} \rightarrow 0$ as $h, k \rightarrow 0$.

\subsubsection{Stability of CN}

Stability of the associated finite difference Equation (36), which is in linear form,

$$
\left.\begin{array}{l}
\left(u_{m}^{n+1}-\frac{1}{2} \mathbf{R} \delta_{x}^{2} u_{m}^{n+1}\right)=\left(u_{m}^{n}+\frac{1}{2} \mathbf{R} \delta_{x}^{2} u^{n}\right)+\frac{1}{2} \mathbf{Q}\left(u_{m}^{n+1}+u_{m}^{n}\right) \mathbf{A} \\
\left(v_{m}^{n+1}-\frac{1}{2} \mathbf{R} \delta_{x}^{2} v_{m}^{n+1}\right)=\left(v_{m}^{n}+\frac{1}{2} \mathbf{R} \delta_{x}^{2} v^{n}\right)+\frac{1}{2} \mathbf{Q}\left(v_{m}^{n+1}+v_{m}^{n}\right) \mathbf{A}
\end{array}\right\}
$$

where $\mathbf{R}, \mathbf{Q}$ and $\mathbf{A}$ are mentioned in Equation (16) and Equation (27) respectively. From Equation (43), we can write, 


$$
\left[\begin{array}{cc}
c_{1} & 0 \\
0 & c_{1}
\end{array}\right]\left[\begin{array}{l}
u^{n+1} \\
v^{n+1}
\end{array}\right]=\left[\begin{array}{cc}
c_{2} & 0 \\
0 & c_{2}
\end{array}\right]\left[\begin{array}{l}
u^{n} \\
v^{n}
\end{array}\right]
$$

where $c_{1}=1+2 \mathbf{R} \sin ^{2}(\beta h / 2)-\frac{1}{2} \mathbf{A Q}$ and $c_{2}=1-2 \mathbf{R} \sin ^{2}(\beta h / 2)+\frac{1}{2} \mathbf{A Q}$.

According to Von-Neumann stability analysis, we have

$$
\psi^{n+1}=G \psi^{n}
$$

where

$$
G=\left[\begin{array}{ll}
c_{1} & 0 \\
0 & c_{1}
\end{array}\right]^{-1}\left[\begin{array}{cc}
c_{2} & 0 \\
0 & c_{2}
\end{array}\right]
$$

where matrix $G$ is called the amplification matrix. According to Von-Neumann stability analysis (29). We have

$$
\Rightarrow \frac{\mid \frac{\left|c_{2}\right|}{\left|c_{1}\right|} \leq 1}{\left|1-2 \mathbf{R} \sin ^{2}(\beta h / 2)+\frac{1}{2} \mathbf{A Q}\right|} \leq 1
$$

Above Equation (46), satisfies the Von-Neumann stability criterion, which shows that CN scheme for Equation (5) is unconditionally stable [21] [22] [23] [24] [25].

\subsection{Fourth Order Accurate Implicit Scheme}

Let us apply another implicit scheme with improved accuracy in space to Equation (5).

$$
\left.\begin{array}{l}
\frac{u_{i}^{n+1}-u_{i}^{n}}{k}=\frac{\beta_{1}}{h^{2}}\left[1+\delta_{x}^{2}\right]^{-1}\left(u_{i}^{n+1}+u_{i}^{n}\right)+\alpha_{1}\left[1+\delta_{x}^{2}\right]^{-1} u_{i}^{n}\left(1-u_{i}^{n}\right)\left(1-v_{i}^{n}\right) \\
\left.\begin{array}{l}
\frac{v_{i}^{n+1}-v_{i}^{n}}{k}=\frac{\beta_{2}}{h^{2}}\left[1+\delta_{x}^{2}\right]^{-1}\left(v_{i}^{n+1}+v_{i}^{n}\right)+\alpha_{2}\left[1+\delta_{x}^{2}\right]^{-1} v_{i}^{n}\left(1-v_{i}^{n}\right)\left(1-u_{i}^{n}\right)
\end{array}\right\} \\
\left(u_{i}^{n+1}-u_{i}^{n}\right)\left[1+\delta_{x}^{2}\right]=\frac{\mathbf{R}_{1}}{2}\left(u_{i}^{n+1}+u_{i}^{n}\right)+\mathbf{Q}_{1}\left[1+\delta_{x}^{2}\right] u_{i}^{n}\left(1-u_{i}^{n}\right)\left(1-v_{i}^{n}\right) \\
\left.\left(v_{i}^{n+1}-v_{i}^{n}\right)\right)\left[1+\delta_{x}^{2}\right]=\frac{\mathbf{R}_{2}}{2}\left(v_{i}^{n+1}+v_{i}^{n}\right)+\mathbf{Q}_{2}\left[1+\delta_{x}^{2}\right] v_{i}^{n}\left(1-v_{i}^{n}\right)\left(1-u_{i}^{n}\right)
\end{array}\right\}
$$

where $\mathbf{R}_{1}, \mathbf{R}_{2}$, and $\mathbf{Q}_{1}, \mathbf{Q}_{2}$ are mentioned in Equation (16).

$$
\begin{aligned}
& a_{1} u_{i}^{n+1}-a_{2} u_{i}^{n}+a_{3}\left(u_{i+1}^{n+1}+u_{i-1}^{n+1}\right)+a_{4}\left(u_{i+1}^{n}-u_{i-1}^{n}\right) \\
& -\frac{1}{2} \mathbf{Q}_{1} \mathbf{P}\left[\frac{5}{6}\left(u_{i}^{n+1}+u_{i}^{n}\right)+\frac{1}{12}\left(u_{i+1}^{n+1}+u_{i-1}^{n+1}+u_{i+1}^{n}+u_{i-1}^{n}\right)\right] \\
& a_{1} v_{i}^{n+1}-a_{2} v_{i}^{n}+a_{3}\left(v_{i+1}^{n+1}+v_{i-1}^{n+1}\right)+a_{4}\left(v_{i+1}^{n}-v_{i-1}^{n}\right) \\
& -\frac{1}{2} \mathbf{Q}_{1} \mathbf{P}\left[\frac{5}{6}\left(v_{i}^{n+1}+v_{i}^{n}\right)+\frac{1}{12}\left(v_{i+1}^{n+1}+v_{i-1}^{n+1}+v_{i+1}^{n}+v_{i-1}^{n}\right)\right]
\end{aligned}
$$

combine Equations (49) (50), to get the following, 


$$
\left.\begin{array}{l}
a_{1} u_{i}^{n+1}-a_{2} u_{i}^{n}+a_{3}\left(u_{i+1}^{n+1}+u_{i-1}^{n+1}\right)+a_{4}\left(u_{i+1}^{n}-u_{i-1}^{n}\right) \\
-\frac{1}{2} \mathbf{Q}_{1} \mathbf{P}\left[\frac{5}{6}\left(u_{i}^{n+1}+u_{i}^{n}\right)+\frac{1}{12}\left(u_{i+1}^{n+1}+u_{i-1}^{n+1}+u_{i+1}^{n}+u_{i-1}^{n}\right)\right] \\
a_{1} v_{i}^{n+1}-a_{2} v_{i}^{n}+a_{3}\left(v_{i+1}^{n+1}+v_{i-1}^{n+1}\right)+a_{4}\left(v_{i+1}^{n}-v_{i-1}^{n}\right) \\
-\frac{1}{2} \mathbf{Q}_{1} \mathbf{P}\left[\frac{5}{6}\left(v_{i}^{n+1}+v_{i}^{n}\right)+\frac{1}{12}\left(v_{i+1}^{n+1}+v_{i-1}^{n+1}+v_{i+1}^{n}+v_{i-1}^{n}\right)\right]
\end{array}\right\}
$$

where $a_{1}=\frac{5}{6}+\mathbf{R}_{1}, a_{2}=\frac{5}{6}-\mathbf{R}_{1}, a_{3}=\frac{1}{2}\left(\frac{1}{6}-\mathbf{R}_{1}\right), a_{4}=\frac{1}{2}\left(\frac{1}{6}+\mathbf{R}_{1}\right)$, and

$$
\mathbf{P}_{m}^{n}=\left(1-\frac{1}{2}\left(u_{i}^{n+1}+u_{i}^{n}\right)\right)\left(1-\frac{1}{2}\left(v_{i}^{n+1}+v_{i}^{n}\right)\right)
$$

\subsubsection{Accuracy of Douglas Scheme}

Accuracy of the Douglas scheme to Equation (36), we apply Taylor's series on each term. After some simplification, resultant is as follows,

$$
\begin{aligned}
u_{\text {Resultant }}= & \left(u_{t}-u_{x x}-u(1-u)(1-v)\right) k+\frac{1}{2} k^{2}\left[u_{t t}-u_{x x t}+u v_{t}(1-u)-u_{t}(1-u)\right. \\
& \left.\times(1-v)+u u_{t}(1-v)\right]+\frac{1}{2}\left[u_{x x t}-u_{x x x x}+u_{x x}(1-u)(1-v)\right] k h^{2} \\
& +\frac{1}{24} h^{2} k^{2}\left[u_{t t x x}-u_{x x x x t}+u_{x x} v_{t}(1-u)-u_{x x} u_{t}(1-u)(1-v)\right. \\
+ & \left.(1-u) u_{x x t}(1-v)\right]-\frac{1}{12} k h^{2} u_{x x x x}+k^{3}\left(\frac{1}{6} u_{t t t}-\frac{1}{4} u_{x x t t}+\cdots\right) \\
& -\frac{1}{144} k h^{4}\left[u_{x x x x t}-\frac{1}{360} u_{x x x x x x}+\frac{1}{144}(1-u)(1-v) u_{x x x x}\right]+\cdots \\
v_{\text {Resultant }}= & \left(v_{t}-v_{x x}-v(1-v)(1-u)\right) k+\frac{1}{2} k^{2}\left[v_{t t}-v_{x x t}+v u_{t}(1-v)-v_{t}(1-v)\right. \\
& \left.\times(1-u)+v v_{t}(1-u)\right]+\frac{1}{2}\left[v_{x x t}-v_{x x x x}+v_{x x}(1-v)(1-u)\right] k h^{2} \\
& +\frac{1}{24} h^{2} k^{2}\left[v_{t t x x}-v_{x x x x t}+v_{x x} v_{t}(1-v)-v_{x x} v_{t}(1-v)(1-u)\right. \\
& \left.+(1-v) v_{x x t}(1-u)\right]-\frac{1}{12} k h^{2} v_{x x x x}+k^{3}\left(\frac{1}{6} v_{t t t}-\frac{1}{4} v_{x x t t}+\cdots\right) \\
& -\frac{1}{144} k h^{4}\left[v_{x x x x t}-\frac{1}{360} v_{x x x x x x}+\frac{1}{144}(1-v)(1-u) v_{x x x x}\right]+\cdots \\
& \quad-\frac{1}{12} k h^{2} v_{x x x x}+k^{3}\left(\frac{1}{6} v_{t t t}-\frac{1}{4} v_{x x t t}+\cdots\right)+\cdots \\
u_{\text {Resultant }}= & \frac{1}{2}\left[u_{x x t}-u_{x x x x}+u_{x x}(1-u)(1-v)\right] k h^{2}+\frac{1}{24} h^{2} k^{2}\left[u_{t t x x}-u_{x x x x t}\right. \\
& \left.+u_{x x} v_{t}(1-u)-u_{x x} u_{t}(1-u)(1-v)+(1-u) u_{x x t}(1-v)\right] \\
& \quad-\frac{1}{12} k h^{2} u_{x x x x}+k^{3}\left(\frac{1}{6} u_{t t t}-\frac{1}{4} u_{x x t t}+\cdots\right)-\cdots \\
v_{\text {Resultant }}= & \frac{1}{2}\left[v_{x x t}-v_{x x x x}+v_{x x}(1-v)(1-u)\right] k h^{2}+\frac{1}{24} h^{2} k^{2}\left[v_{t t x x}-v_{x x x x t}\right. \\
& \left.+v_{x x} v_{t}(1-v)-v_{x x} v_{t}(1-v)(1-u)+(1-v) v_{x x t}(1-u)\right] \\
& =(5)
\end{aligned}
$$


Dividing above Equations (54) and (55) by $\mathrm{k}$ and take into account Equation (5), so resultants are as follows:

$$
u_{\text {Resultant }}=k^{2}\left(\frac{1}{6} u_{t t t}-\frac{1}{4} u_{x x t t}+\cdots\right)-\frac{1}{144} h^{4}\left[u_{x x x x t}-\cdots\right]+\cdots
$$

let us look above equation in new way,

$$
\left.\begin{array}{l}
u_{\text {Resultant }}=k^{2}\left(\frac{1}{6} u_{t t t}-\frac{1}{4} u_{x x t t}+\cdots\right)-\frac{1}{144} h^{4}\left[u_{x x x x t}-\frac{1}{360} u_{x x x x x x}+\frac{1}{144}(1-u)(1-v) u_{x x x x}\right]+\cdots \\
v_{\text {Resultant }}=k^{2}\left(\frac{1}{6} v_{t t t}-\frac{1}{4} v_{x x t t}+\cdots\right)-\frac{1}{144} h^{4}\left[v_{x x x x t}-\frac{1}{360} v_{x x x x x x}+\frac{1}{144}(1-v)(1-u) v_{x x x x}\right]+\cdots
\end{array}\right\}
$$

Now principle part of the truncation error (PPTE) is as follows:

$$
\left.\begin{array}{l}
P P T E_{u}=-\frac{1}{144} h^{4}\left[u_{x x x x t}+\frac{1}{144}(1-u)(1-v) u_{x x x x}\right]+k^{2}\left(\frac{1}{6} u_{t t t}-\frac{1}{4} u_{x x t t}+\cdots\right)+\cdots \\
P P T E_{v}=-\frac{1}{144} h^{4}\left[v_{x x x x t}+\frac{1}{144}(1-v)(1-u) v_{x x x x}\right]+k^{2}\left(\frac{1}{6} v_{t t t}-\frac{1}{4} v_{x x t t}+\cdots\right)+\cdots
\end{array}\right\}
$$

which shows that this scheme is 4 th order accurate in space, such as $O\left(k^{2}, h^{4}\right)$ [26] [27] [28] [29] [30].

\subsubsection{Consistency of Douglas Scheme}

From accuracy, we find principle part of the truncation error along with Equation (58). Which shows that Douglas scheme is consistent because $z_{m}^{n}=$ PPTE tends to zero as $h$ and $\mathrm{k}$ tends to zero, i.e. $z_{m}^{n} \rightarrow 0$ as $h, k \rightarrow 0 \quad$ [31] [32].

\subsubsection{Stability of Douglas Scheme}

Stability of the associated finite difference Equation (51), which is in linear form, is

$$
\left.\begin{array}{c}
\left(u_{i}^{n+1}-u_{i}^{n}\right)\left[1+\delta_{x}^{2}\right]=\frac{\mathbf{R}}{2} \delta_{x}^{2}\left(u_{i}^{n+1}+u_{i}^{n}\right)+\frac{1}{2} \mathbf{A} \mathbf{Q}\left[1+\delta_{x}^{2}\right]\left(u_{i}^{n+1}+u_{i}^{n}\right) \\
\left(v_{i}^{n+1}-v_{i}^{n}\right)\left[1+\delta_{x}^{2}\right]=\frac{\mathbf{R}}{2} \delta_{x}^{2}\left(v_{i}^{n+1}+v_{i}^{n}\right)+\frac{1}{2} \mathbf{A} \mathbf{Q}\left[1+\delta_{x}^{2}\right]\left(v_{i}^{n+1}+v_{i}^{n}\right)
\end{array}\right\}
$$

Consider Equations (44) (60), in the following few important steps.

$$
\left.\begin{array}{l}
c_{1}=1-\frac{1}{3} \sin ^{2}(\beta h / 2)+2 \mathbf{R} \sin ^{2}(\beta h / 2)-\frac{1}{2} \mathbf{A} \mathbf{Q}+\frac{1}{3} \sin ^{2}(\beta h / 2) \\
c_{2}=1-\frac{1}{3} \sin ^{2}(\beta h / 2)-2 \mathbf{R} \sin ^{2}(\beta h / 2)+\frac{1}{2} \mathbf{A Q}-\frac{1}{3} \sin ^{2}(\beta h / 2)
\end{array}\right\}
$$

Apply Von-Neumann stability analysis to Equation (60), we get the following 


$$
\begin{gathered}
\frac{\left|c_{2}\right|}{\left|c_{1}\right|} \leq 1 \\
\Rightarrow \frac{\left|1-\frac{1}{3} \sin ^{2}(\beta h / 2)-2 \mathbf{R} \sin ^{2}(\beta h / 2)+\frac{1}{2} \mathbf{A Q}-\frac{1}{3} \sin ^{2}(\beta h / 2)\right|}{\left|1-\frac{1}{3} \sin ^{2}(\beta h / 2)+2 \mathbf{R} \sin ^{2}(\beta h / 2)-\frac{1}{2} \mathbf{A Q}+\frac{1}{3} \sin ^{2}(\beta h / 2)\right|} \leq 1 .
\end{gathered}
$$

Above Equation (63), satisfies the Von-Neumann stability criterion, which shows that fourth order implicit scheme is unconditionally stable [33] [34] [35] [36] [37].

\subsection{Richardson Extrapolation Technique}

Richardson extrapolation method lead to considerable improvement of numerical results which solving the partial differential equation system by finite difference method. Richardson's extrapolation formulae are [38] [39],

$$
N_{2}(h)=N_{(1)}\left(\frac{h}{2}\right)+\frac{N_{(1)}\left(\frac{h}{2}\right)-N_{(1)} h}{4^{(1)}-1},
$$

above formula leads to get fourth order accuracy [39] [40],

$$
N_{2}(h)=N_{(1)}\left(\frac{h}{4}\right)+\frac{N_{(1)}\left(\frac{h}{4}\right)-N_{(1)}\left(\frac{h}{2}\right)}{3}
$$

\section{Error Norms}

The aim of the accuracy is assessed by some redefined norms, associated with the consistency of the finite difference schemes, such scaled measurement to error defined in term of norms specially $L_{2}$ and $L_{\infty}$, which are outlined below:

$$
\begin{gathered}
L_{2}=\sqrt{\sum_{i=0}^{N}\left|u_{i}^{\text {Exact }}-u_{i}^{\text {Approximation }}\right|^{2}} \\
L_{\infty}=\max _{i}\left|u_{i}^{\text {Exact }}-u_{i}^{\text {Approximation }}\right| \\
L_{\text {Relative }}=\frac{L_{2}}{\sqrt{\sum_{i=0}^{N}\left|u_{i}^{\text {Exact }}\right|^{2}}} \\
\text { Absolute }_{\text {Error }}=\left|u_{i}^{\text {Exact }}-u_{i}^{\text {Approximation }}\right| \\
\text { Rate of Convergence }=\frac{\log \left(E_{h_{1}} / E_{h_{2}}\right)}{\log \left(h_{1} / h_{2}\right)}
\end{gathered}
$$

where $E_{h_{1}}$ and $E_{h_{2}}$ are errors in $L_{2}$ and $L_{\infty}$ at number of partitions $h$ and $\mathrm{h} / 2$ respectively [41] [42].

\section{Results}

Numerical computations have been performed using the uniform grid [14] [21] 
[25] [29]. For the test problem (5), we analysed results with various finite difference schemes. In Tables 1-3, we fixed domain as $-8 \leq x \leq 8$ with $k=$ 0.0001 and find results at $t=0.5$. In these three tables, we obtain insight error. Table 4 and Table 5 show results for $L_{2}, L_{\infty}$ and Error $_{\text {Relative }}$ norms for major

Table 1. Estimates of results using Crank Nicolson Implicit Scheme. Fixed some parameters such as $k=0.0001, t=0.5$, Grid $=(36 \times 36)$ and $[a, b]=\left[\begin{array}{ll}-8 & 8\end{array}\right]$. Error means simple absolute error for two components $u$ and $v$ in different columns.

\begin{tabular}{cccccccc}
\hline time $=t$ & $X$ & $u_{A p p .}$ & $U_{E x .}$ & Error & $V_{A p p .}$ & $V_{E x .}$ & Error \\
\hline 0.5 & -7.6 & 0.0046011 & 0.0041001 & 0.000501 & 0.9953994 & 0.9952149 & 0.000184 \\
& -3.2 & 0.0085027 & 0.0083021 & 0.000200 & 0.9914981 & 0.9911982 & 0.000299 \\
& -1.6 & 0.0096107 & 0.0092104 & 0.000401 & 0.9903902 & 0.9900162 & 0.000374 \\
-0.4 & 0.0176852 & 0.01761453 & 0.000071 & 0.9823165 & 0.9821154 & 0.000202 \\
2 & 0.0975382 & 0.0972546 & 0.00013 & 0.9024705 & 0.9020103 & 0.000611 \\
4 & 0.4845664 & 0.4841066 & 0.00046 & 0.5154585 & 0.5152934 & 0.000160 \\
5.6 & 0.7288725 & 0.7281615 & 0.00071 & 0.2711472 & 0.2710380 & 0.000109 \\
8.4 & 0.8968938 & 0.8965883 & 0.00031 & 0.1031154 & 0.1030094 & 0.000106 \\
\hline
\end{tabular}

Table 2. Estimates of results using Fourth Order Implicit Scheme. Fixed some parameters such as $k=0.0001, t=0.5$, Grid $=(36 \times 36)$ and $[a, b]=[-88]$. Error means simple absolute error for two components $u$ and $v$ in different columns.

\begin{tabular}{cccccccc}
\hline time $=t$ & $x$ & $u_{\text {App. }}$ & $U_{\text {Ex. }}$ & Error & $V_{\text {App. }}$ & $V_{\text {Ex. }}$ & Error \\
\hline 0.5 & -7.6 & 0.0041091 & 0.0041001 & 0.000009 & 0.9952994 & 0.9952149 & 0.0000845 \\
& -3.2 & 0.0083067 & 0.0083021 & 0.000021 & 0.9911981 & 0.9911001 & 0.0000981 \\
& -1.6 & 0.0092145 & 0.0092104 & 0.000050 & 0.9900902 & 0.9900162 & 0.0000742 \\
-0.4 & 0.0176109 & 0.0176141 & 0.000011 & 0.9821165 & 0.9821154 & 0.0000220 \\
2 & 00.0972592 & 0.0972546 & 0.000017 & 0.9020705 & 0.9020103 & 0.0000601 \\
4 & 0.4841021 & 0.4841066 & 0.000060 & 0.5152585 & 0.5152934 & 0.0000351 \\
5.6 & 0.7281665 & 0.7281615 & 0.000011 & 0.2710472 & 0.2710380 & 0.0000923 \\
& 8.4 & 0.8965813 & 0.8965883 & 0.000005 & 0.1030154 & 0.1030094 & 0.000006 \\
\hline
\end{tabular}

Table 3. Estimates of results using Sixth Order Extrapolation Method. Fixed some parameters such as $k=0.0001, t=0.5$, Grid $=(36 \times 36)$ and $[a, b]=[-88]$. Error means simple absolute error for two components $u$ and $v$ in different columns.

\begin{tabular}{|c|c|c|c|c|c|c|c|}
\hline Time $=t$ & $x$ & $u_{\text {App. }}$ & $U_{E x .}$ & Error & $v_{\text {App. }}$ & $V_{E x .}$ & Error \\
\hline \multirow[t]{8}{*}{0.5} & -7.6 & 0.0041211 & 0.0041001 & 0.0000021 & 0.9952117 & 0.9952149 & 0.00000032 \\
\hline & -3.2 & 0.0083227 & 0.0083021 & 0.0000021 & 0.9911012 & 0.9911001 & 0.00000011 \\
\hline & -1.6 & 0.0092607 & 0.0092104 & 0.0000053 & 0.9900121 & 0.9900162 & 0.00000041 \\
\hline & -0.4 & 0.0176152 & 0.0176141 & 0.0000011 & 0.9821116 & 0.9821154 & 0.00000038 \\
\hline & 2 & 0.0972382 & 0.0972546 & 0.0000017 & 0.9020100 & 0.9020103 & 0.00000003 \\
\hline & 4 & 0.4841664 & 0.4841066 & 0.0000059 & 0.5152959 & 0.5152934 & 0.00000025 \\
\hline & 5.6 & 0.7281725 & 0.7281615 & 0.0000015 & 0.2710339 & 0.2710380 & 0.0000004 \\
\hline & 8.4 & 0.8965938 & 0.8965883 & 0.000005 & 0.1030091 & 0.1030094 & 0.0000003 \\
\hline
\end{tabular}


Table 4. Estimates of results, with comparison of errors as mentioned in formulae. Fixed some parameters such as $k=0.0001, t=1$, and $[a, b]=\left[\begin{array}{ll}-8 & 8\end{array}\right]$.

\begin{tabular}{|c|c|c|c|c|c|c|c|}
\hline \multirow{2}{*}{$t$} & \multirow{2}{*}{ Grid } & \multicolumn{3}{|c|}{ Crank Nicolson } & \multicolumn{3}{|c|}{ Douglas } \\
\hline & & $L_{2}$ & $L_{\infty}$ & Error $_{\text {relative }}$ & $L_{2}$ & $L_{\infty}$ & Error $_{\text {relative }}$ \\
\hline \multirow[t]{4}{*}{1.0} & $31 \times 31$ & $1.29 e^{-3}$ & $8.79 e^{-4}$ & $1.53 e^{-5}$ & $1.14 e^{-4}$ & $1.14 e^{-5}$ & $4.3 e^{-7}$ \\
\hline & $51 \times 51$ & $1.9 e^{-3}$ & $3.47 e^{-4}$ & $1.36 e^{-6}$ & $1.7 e^{-4}$ & $2.21 e^{-6}$ & $8.7 e^{-8}$ \\
\hline & $81 \times 81$ & $3.24 e^{-4}$ & $1.19 e^{-5}$ & $2.04 e^{-6}$ & $2.0 e^{-5}$ & $2.18 e^{-6}$ & $8.11 e^{-8}$ \\
\hline & $101 \times 101$ & $1.24 e^{-4}$ & $4.91 e^{-6}$ & $1.57 e^{-7}$ & $2.2 e^{-6}$ & $2.83 e^{-7}$ & $9.08 e^{-9}$ \\
\hline
\end{tabular}

Table 5. Estimates of results, with two different Implicit FD schemes. Relative error values give encouragement to our results. Fixed some parameters as Grid $=(51 \times 51), k=$ 0.0001 and $h$ according to grid with wave speed $c=2$ and $z=1$.

\begin{tabular}{ccccccc}
\hline \multirow{2}{*}{$t$} & \multicolumn{3}{c}{ Crank Nicolson } & \multicolumn{3}{c}{ Douglas } \\
\cline { 2 - 7 } & $L_{2}$ & $L_{\infty}$ & Error $_{\text {relative }}$ & $L_{2}$ & $L_{\infty}$ & Error $_{\text {relative }}$ \\
\hline 0.1 & 0.0012 & 0.000031 & 0.0000013 & 0.000067 & 0.0000011 & 0.00000079 \\
0.3 & 0.0019 & 0.000043 & 0.0000027 & 0.000071 & 0.0000019 & 0.00000008 \\
0.5 & 0.00017 & 0.000051 & 0.0000039 & 0.000079 & 0.0000031 & 0.000000081 \\
0.7 & 0.00021 & 0.000079 & 0.0000048 & 0.000081 & 0.0000042 & 0.000000094 \\
\hline
\end{tabular}

schemes $\mathrm{CN}$ and Douglas at different grids and time levels. Rate of convergence is defined in Table 6. All these six tables show results for three implicit scheme to analyse error and performance of the schemes. According to results mentioned in tables, these scheme play very important role for convergence although non-linear system is difficult to stabilized. In Figure 1, FTCS conditionally stable finite difference scheme is used at different grid, also we did in Figure 2 with Crank Nicolson implicit scheme. Fixed some parameters in $\mathrm{CN}$ scheme to get common region at different time level, as we mentioned in Figure 3. In Figure 4 and Figure 5, we did comparison of results with two different schemes at various time levels. In Figure 6 and Figure 7 attractive common region at different time level is obtained. In Figures 8-10, comparison of results with Richardson, Douglas and exact in $u(x, t)$ component and $u(x, t) / v(x, t)$ form. Last Figure 11 is obtained at $u(x, t) / v(x, t)$ form of results with two finite difference schemes. From Tables and Figures, we observed that these schemes exactly predict accuracy as we learn from literature reviews.

\section{Conclusions}

In this chapter, the solution to one dimensional coupled Fisher KPP system is successfully approximated by a various numerical finite difference schemes. Explicit FTCS is conditionally stable, and we give more attention to parameter $\mathbf{R}_{1}$ and $\mathbf{R}_{2}$, which can be used to stabilized the results as we can see from Figure 1. Crank Nicolson and Douglas schemes are implicit schemes with unconditionally stable nature, this guarantee is given by Von-Neumann stability analysis 
Table 6. Estimates the rate of convergence for two implicit FD schemes for different grids.

\begin{tabular}{ccccccc}
\hline \multirow{2}{*}{ Grid } & \multicolumn{3}{c}{ Crank Nicolson } & \multicolumn{3}{c}{ Douglas } \\
\cline { 2 - 7 } & Rate $L_{2}$ & Rate $L_{\infty}$ & Rate $E_{r}$ & $L_{2}$ & $L_{\infty}$ & Error reative \\
\hline $31 \times 31$ & - & - & - & - & - & - \\
$51 \times 51$ & 2.1882 & 3.0712 & 3.1098 & 2.881 & 3.0941 & 3.8172 \\
$81 \times 81$ & 2.1761 & 3.1971 & 3.2910 & 2.5619 & 3.4218 & 3.8971 \\
$101 \times 101$ & 1.4097 & 3.3468 & 4.0118 & 2.9431 & 3.7689 & 3.9981 \\
\hline
\end{tabular}
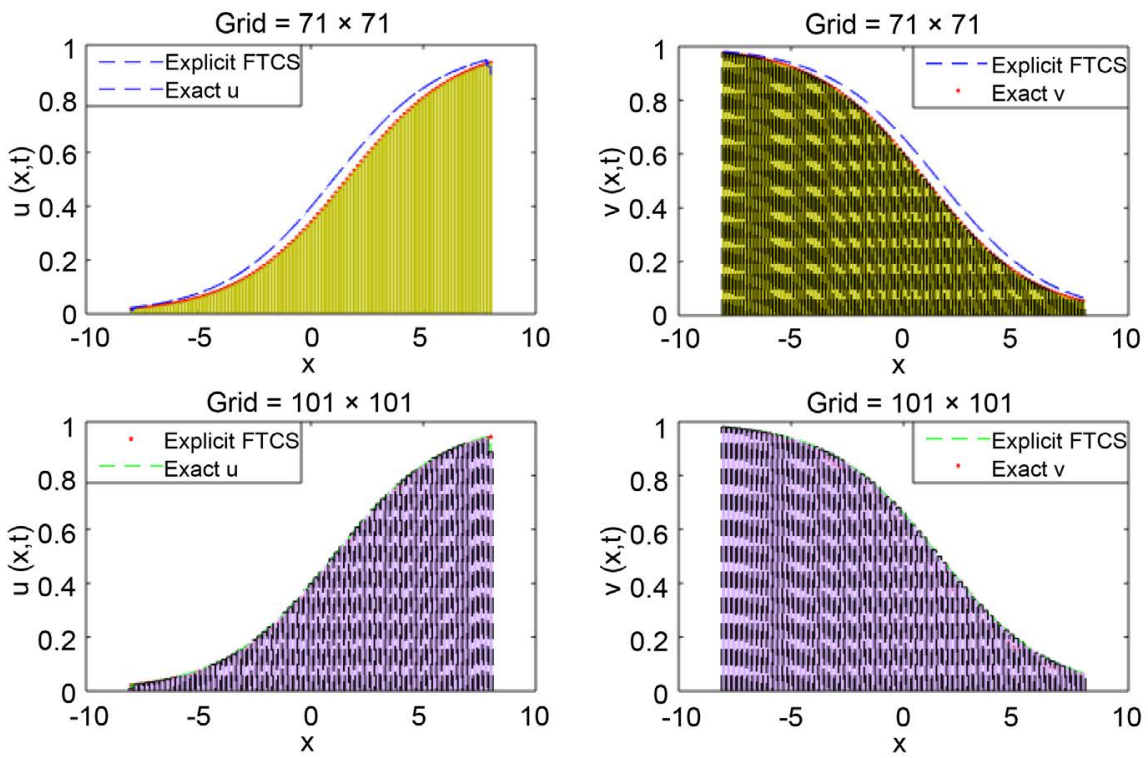

Figure 1. Shows results using FTCS scheme at two different grids, keeping the conditionally stable term. Area under the curves is the common region for two different grids.
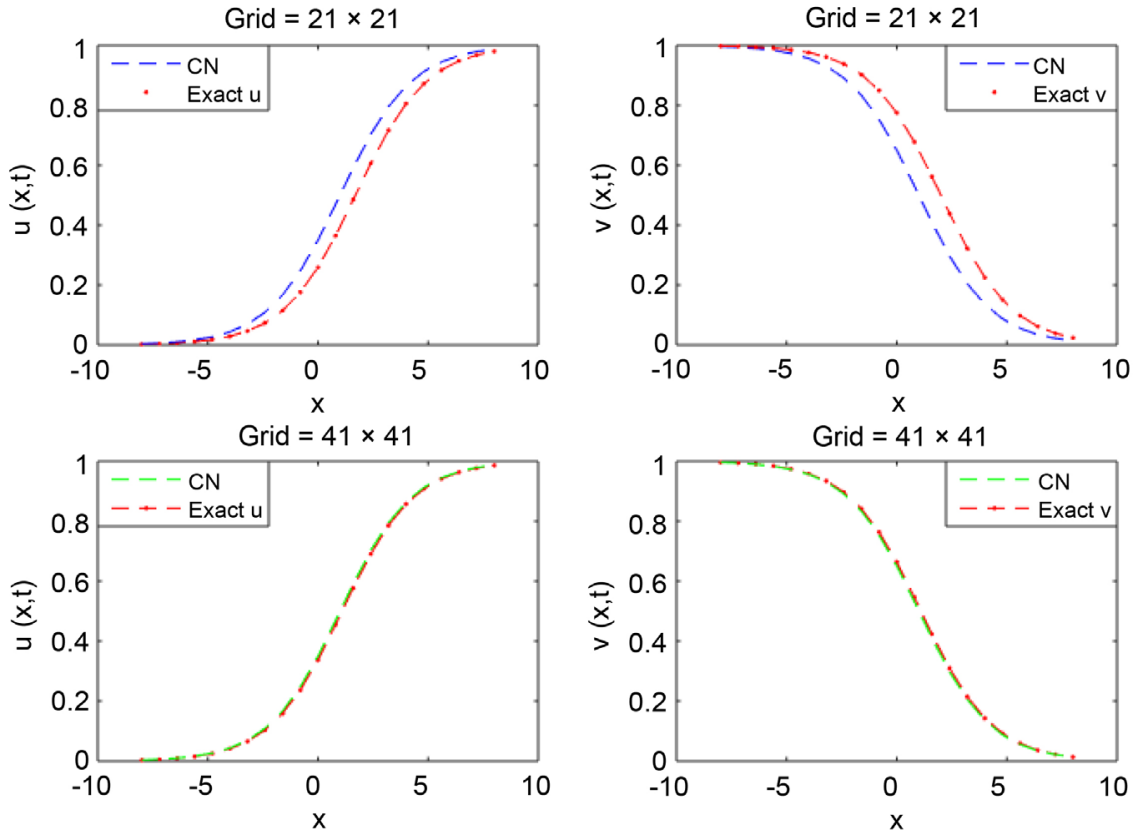

Figure 2. Shows results using Crank Nicolson scheme at two different grids. 

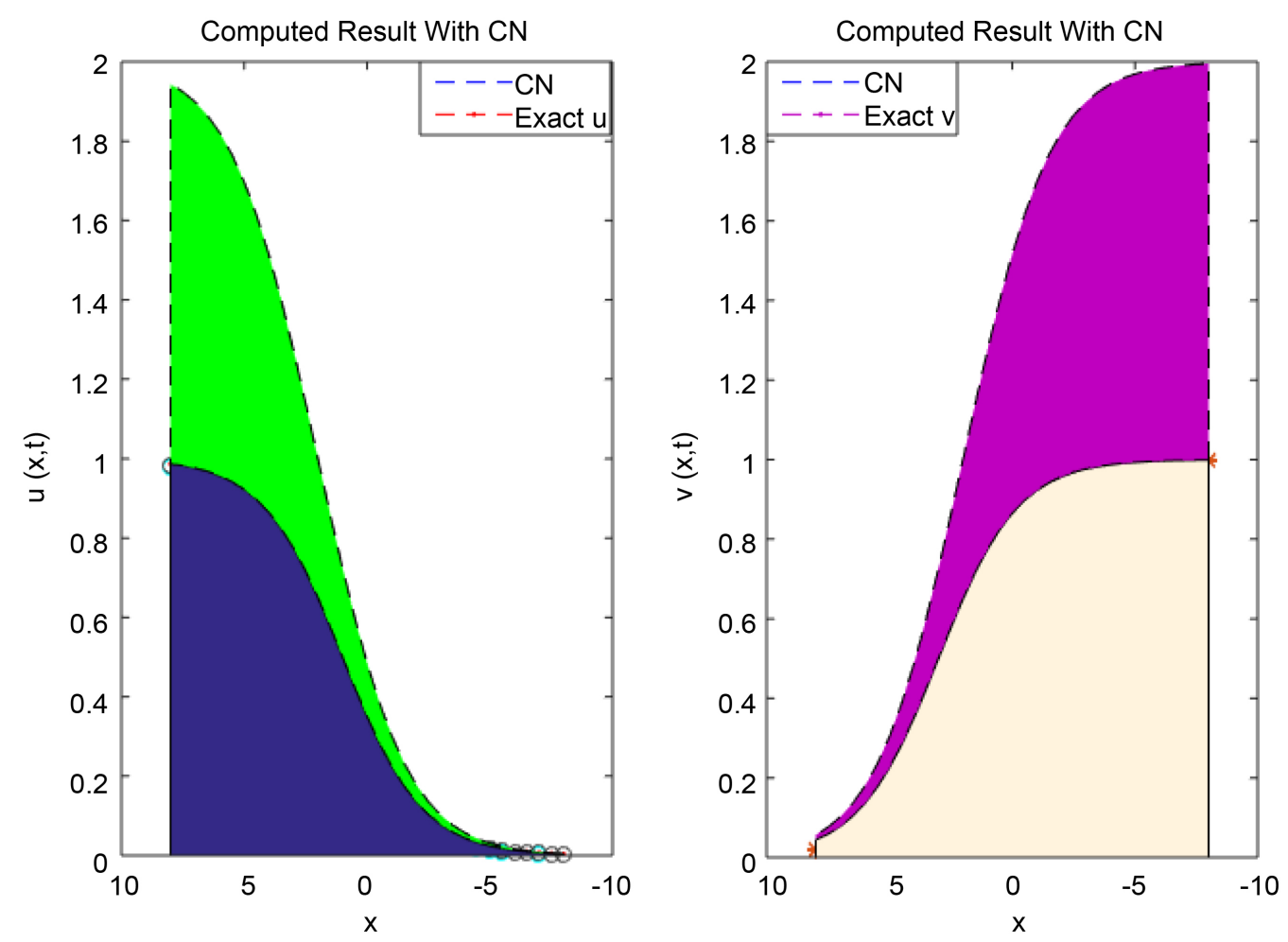

Figure 3. Shows results using Crank Nicolson scheme at two different time level. Common region is the wave front that we study in literature [35] [39] [41].
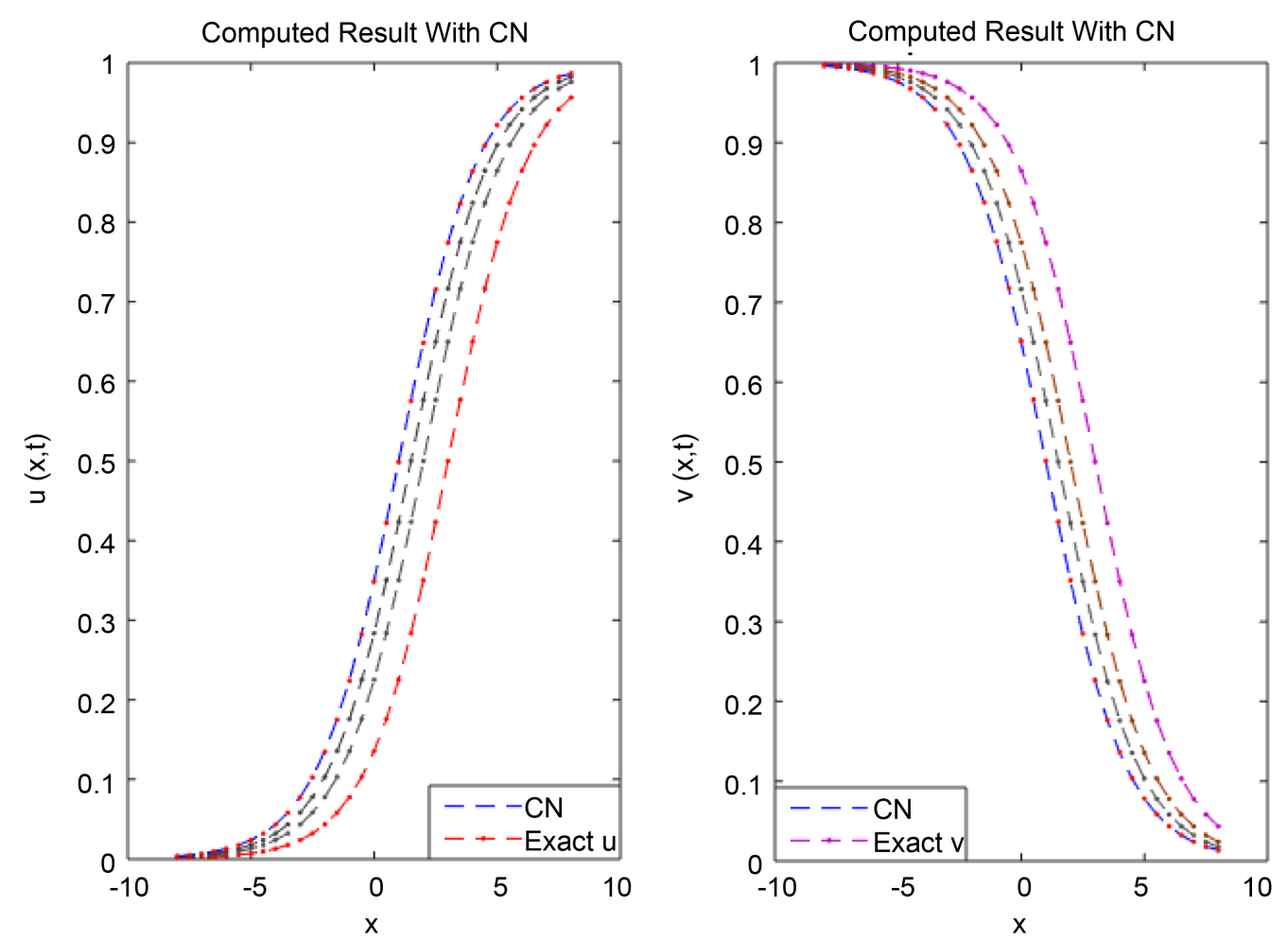

Figure 4. Shows results using Crank Nicolson scheme at various time levels. We did comparison with exact solution for $u(x, t)$ and $v(x, t)$ components. 

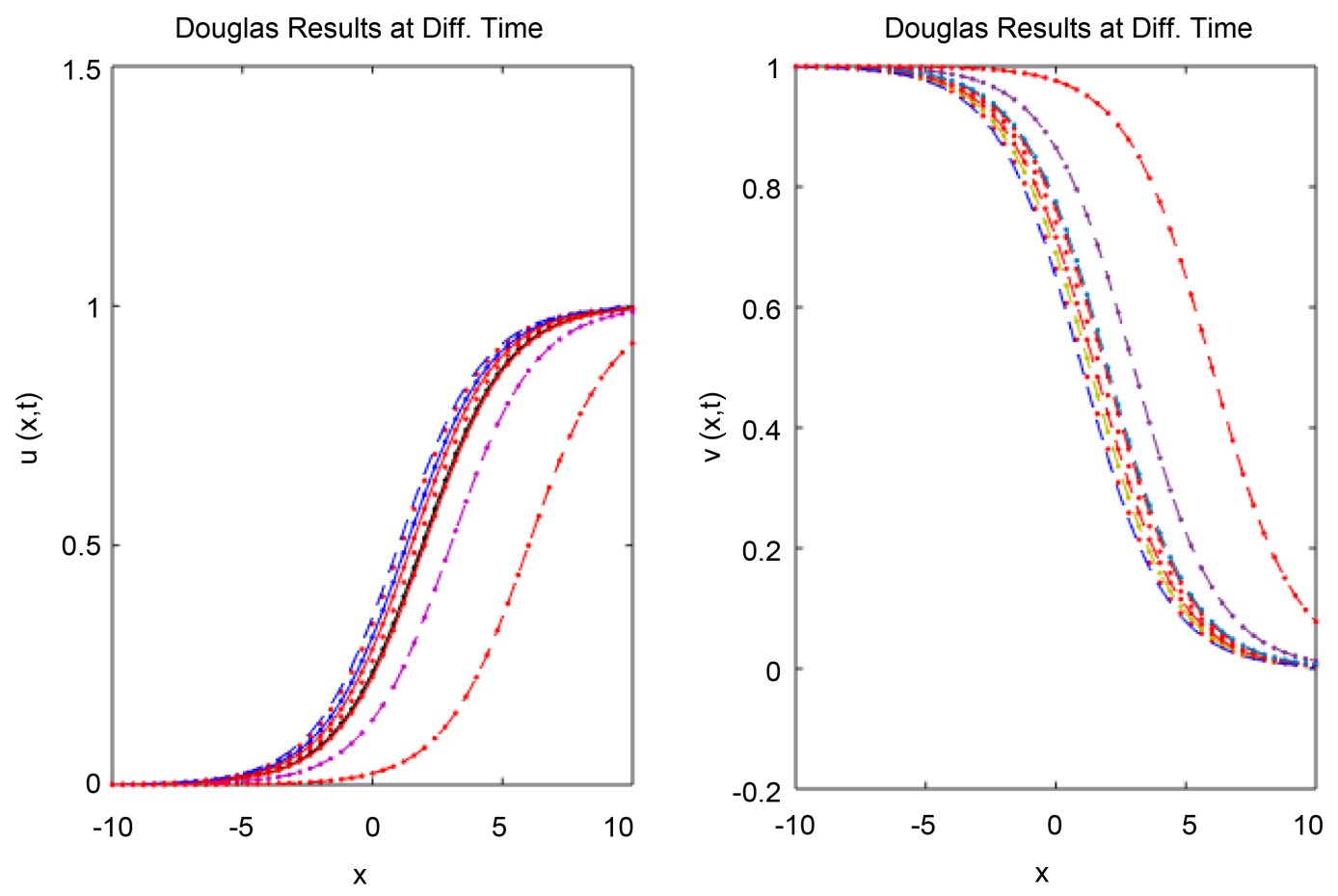

Figure 5. Shows results using fourth order implicit scheme at various time levels. We did comparison with exact solution for $u(x, t)$ and $v(x, t)$ components.
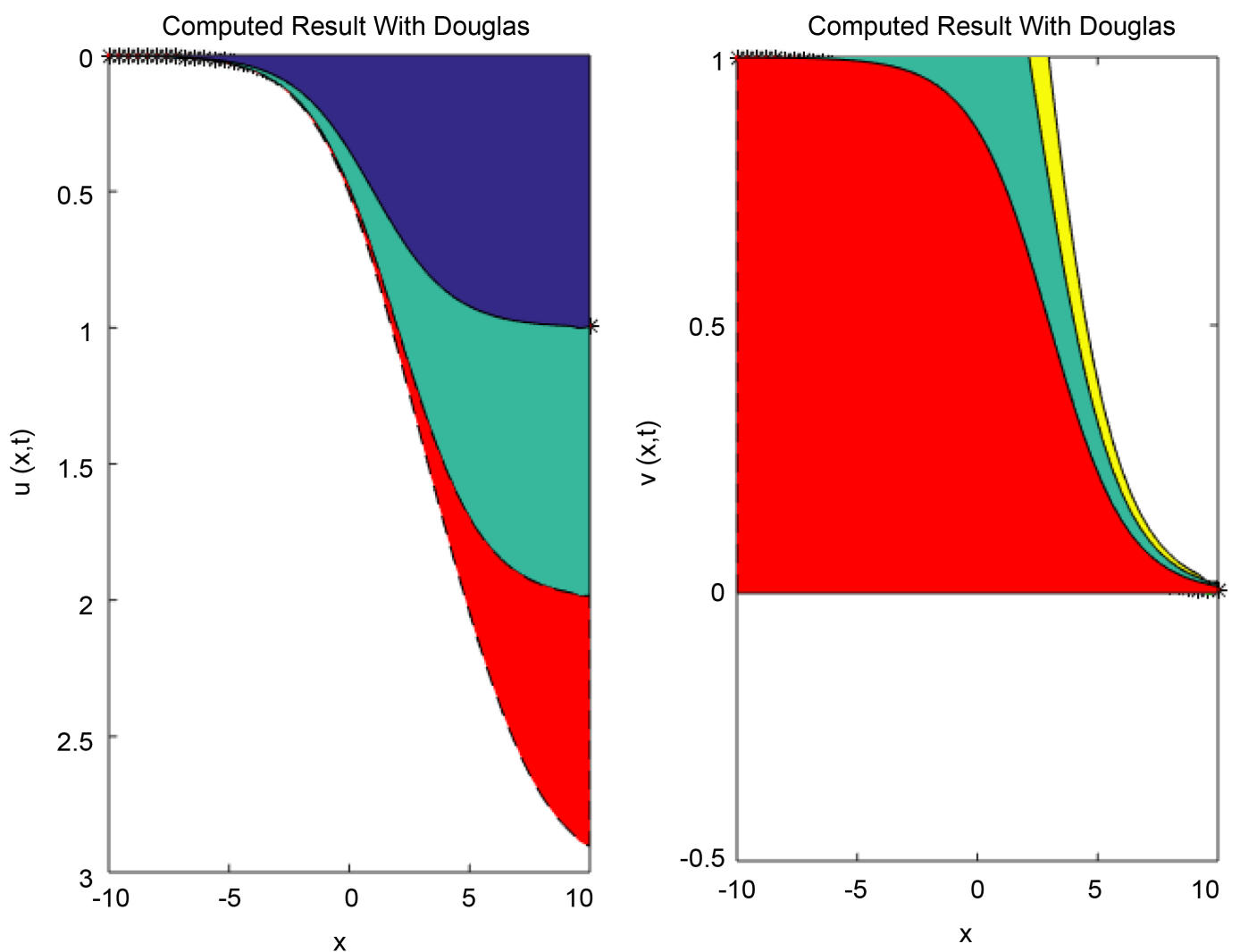

Figure 6. Shows fourth order implicit scheme at three different time level. Common region is the wave front that we study in literature [17] [23] [29] [41]. 

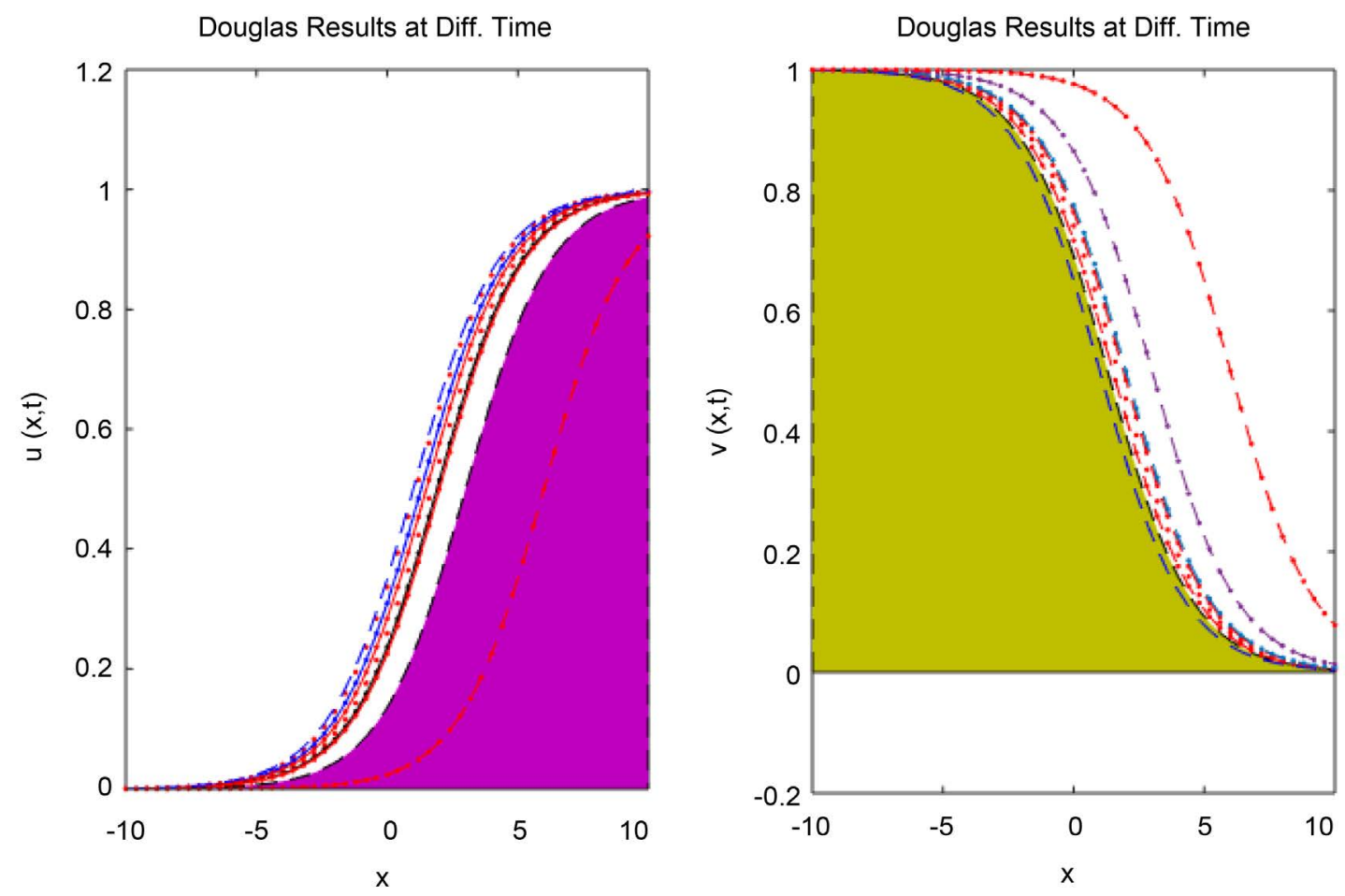

Figure 7. Shows results using fourth order implicit scheme at various time levels, with common region.

Solution with Douglas, Exact, Richardson

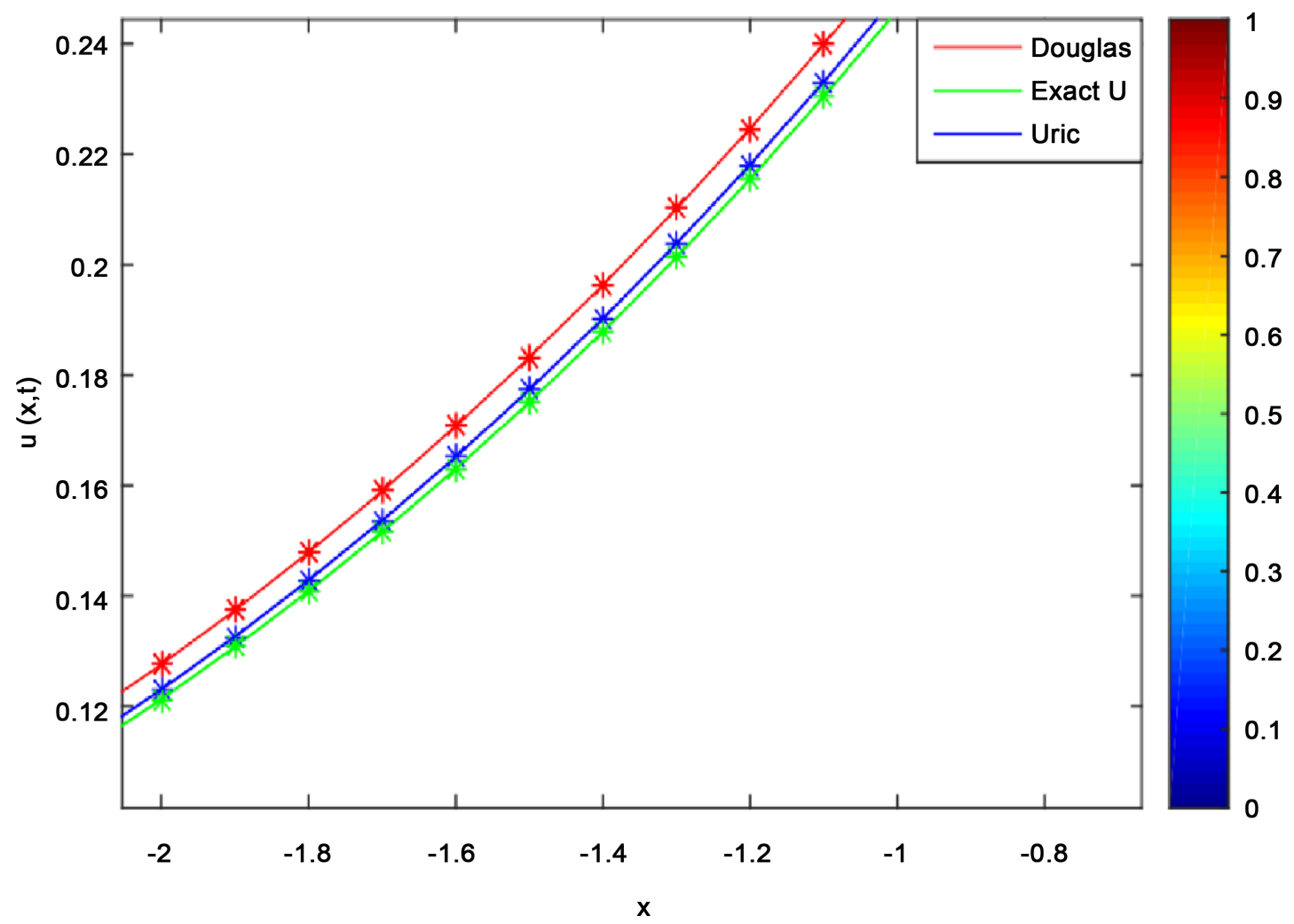

Figure 8. Shows results using Richardson Extrapolation and fourth order implicit scheme, with comparison to exact solution for $\mathrm{u}$ component. 


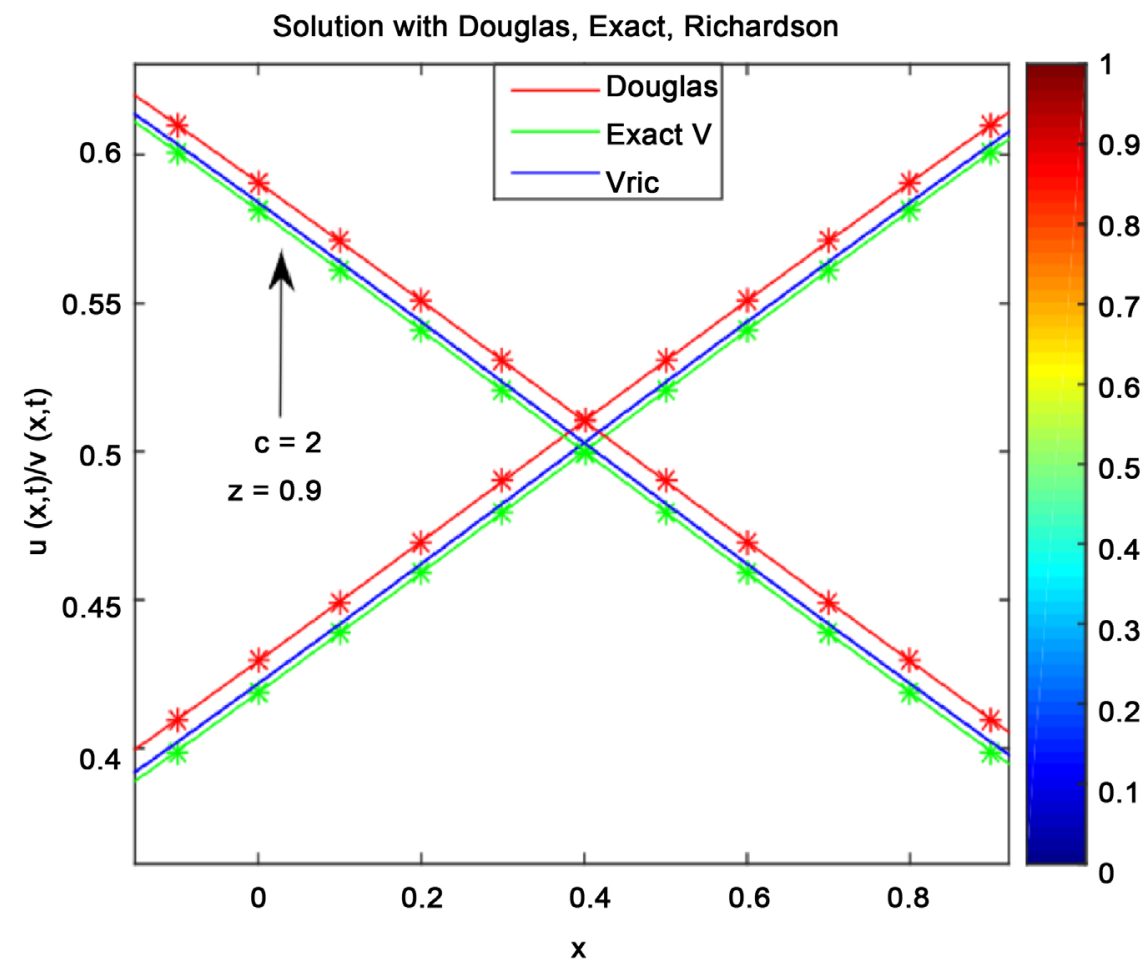

Figure 9. Shows results using Richardson Extrapolation and fourth order implicit scheme, with comparison to exact solution for $u(x, t) / v(x, t)$ form.
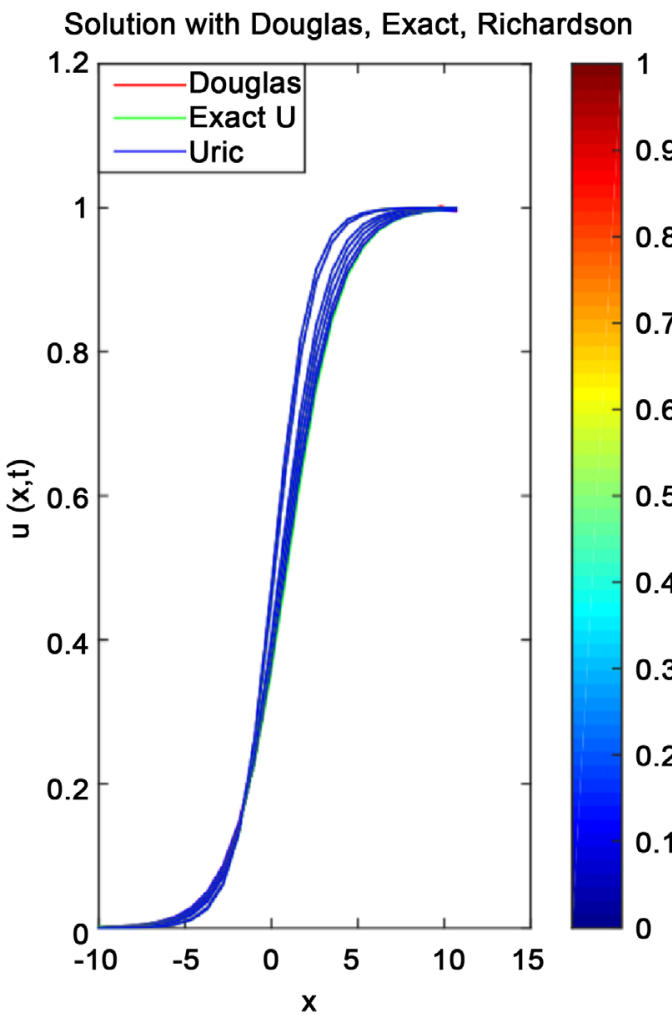

Solution with Douglas, Exact, Richardson

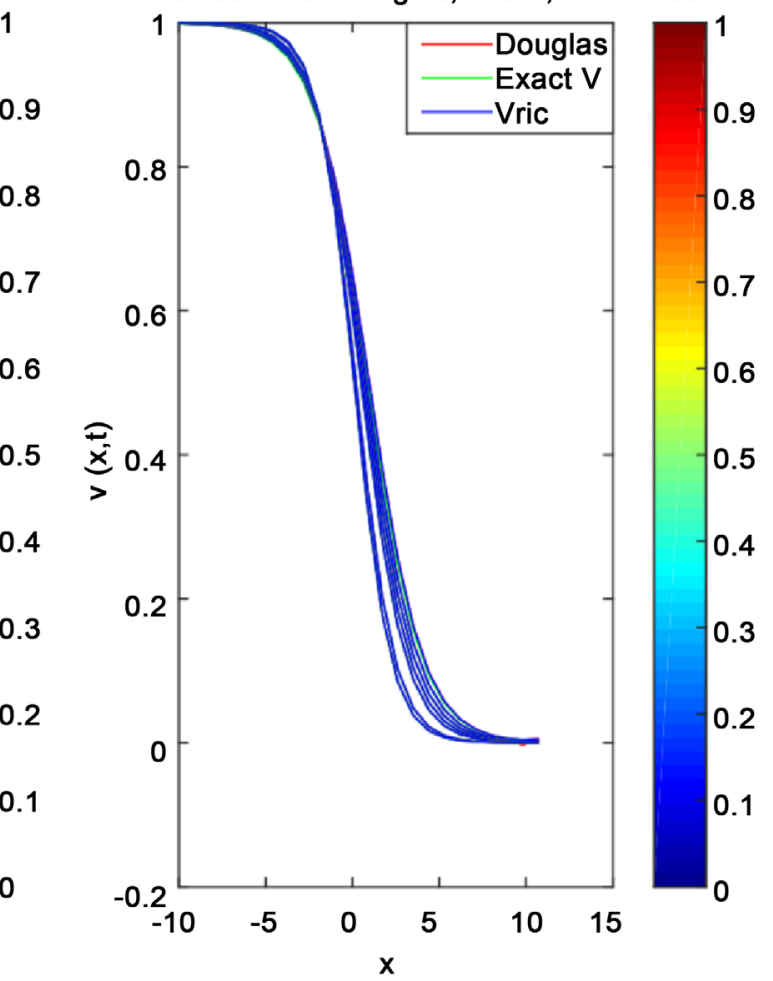

Figure 10. Shows results using Richardson Extrapolation at different time levels, along fourth order implicit scheme. 


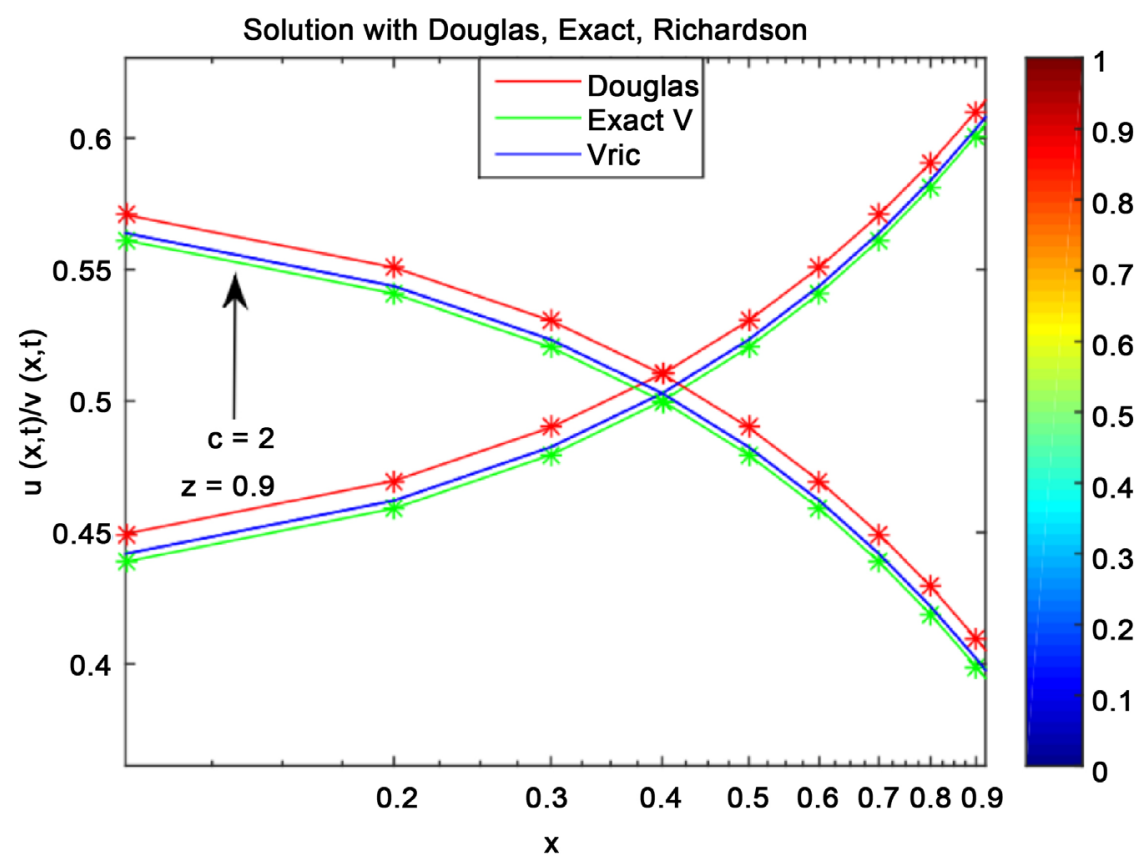

Figure 11. Shows a comparison of two schemes in $u(x, t) / v(x, t)$ form, with exact solution.

as we explained in methodology section [27] [28] [29] [31] [35] [36]. Jacobean found with the help of Newton's iterative method [36] [38], whereas tridiagonal nature of the linear obtained system is solved by Crout's or Partially Pivoting method. These method are highly computable in term of non-linear system as we defined in this chapter [40] [41] [42]. Numerical results show great deal of matchability to exact solution. Accuracy in results are glanced from figures and tables.

\section{Acknowledgements}

Shahid Hasnain, Prof. Daoud Mashat and Muhammad Saqib is thankful to Dr Muhammad Faheem Afzaal, Department of Chemical Engineering, Imperial College London and Vineet K. Srivastava, Scientist, ISTRAC/ISRO, Bangalore, India for thoughtful remarks. This research was supported by Department of Mathematics, division of Numerical Analysis, King Abdulaziz University, Jeddah, Saudi Arabia.

\section{Conflict of Interest}

There is no conflict of interest in this research paper.

\section{References}

[1] Fisher, R.A. (1936) The Wave of Advance of Advantageous Genes. Annals of Eugenics, 7, 355-369. https://doi.org/10.1111/j.1469-1809.1937.tb02153.x

[2] Kolmogorov, A.K., Petrovsky, N.P. and Piscounov, S.P. (1937) Etude de I equations de la diffusion avec croissance de la quantitate de matiere et son application a un probolomebiologique. Bulletin Univercity Mosku, 1, 1-25.

[3] Newman, W.I. (1980) Some Exact Solutions to a Non-Linear Diffusion Problem in 
Population Genetics and Combustion. Journal of Theoretical Biology, 85, 325-334.

[4] Ronson, D.G. and Weinberger, H.F. (1975) Lecture Notes in Mathematics 446, Partial Differential Equations and Related Topics. Springer-Verlag, Berlin, 5-49.

[5] Fahmy, E.S. (2009) Exact Solutions for Some Reaction Diffusion Systems with Nonlinear Reaction Polynomial Terms. Applied Mathematical Sciences, 3, 533-540.

[6] Pao, C.V. (1981) Asymptotic Stability of Reaction-Diffusion Systems in Chemical Reactor and Combustion Theory. Journal of Mathematical Analysis and Applications, 82, 503-526.

[7] Franak Kameneetiskii, D.A. (1969) Diffusion and Heat Transfer in Chemical Kinetics. Plenum Press, New York.

[8] Bramson, M.D. (1978) Maximal Displacement of Branching Brownian Motion. Communications on Pure and Applied Mathematics, 31, 531-581.

https://doi.org/10.1002/cpa.3160310502

[9] Canosa, J.C. (1973) On a Nonlinear Diffusion Equation Describing Population Growth. IBM Journal of Research Development, 17, 307-313. https://doi.org/10.1147/rd.174.0307

[10] Arnold, R.A., Showalter, K. and Tyson, J.J. (1987) Propagation of Chemical Reactions in Space. Journal of Chemical Education, 64, 7740-7744. https://doi.org/10.1021/ed064p740

[11] Tuckwell, H.C. (1988) Introduction to Theoretical Neurobiology. Cambridge University Press, Cambridge.

[12] Mittal, R.C. and Rajni, R.R. () A Study of One Dimensional Nonlinear Diffusion Equations by Bernstein Polynomial Based Differential Quadrature Method. Journal of Mathematical Chemistry, 55, 673-695.

[13] Burgers, J.M. (1948) A Mathematical Model Illustrating the Theory of Turbulence. Advances in Applied Mechanics, 1, 171-199.

[14] Bateman, H.B. (1915) Some Recent Researches on the Motion of Fluids. Monthly Weather Review, 43, 163-170. https://doi.org/10.1175/1520-0493(1915)43<163:SRROTM>2.0.CO;2

[15] Cole, J.D. (1950) On a Quaslinear Parabolic Equations Occurring in Aerodynamics. Applied Mathematics, 9, 201-230.

[16] Hopf, E.H. (1951) The Partial Differential Equation $u_{t}+u u_{x}=\mu u_{x x}$. Communications on Pure and Applied Mathematics, 3, 225-236.

[17] Wang, X.Y. (1988) Exact and Explicit Solitary Wave Solutions for the Generalized Fishers Equation. Physics Letters A, 131, 227-279.

[18] Wazwaz, A.M. and Gorguis, A.G. (2004) An Analytic Study of Fisher's Equation by Using Adomian Decomposition Method. Applied Mathematics and Computation, 154, 609-620.

[19] Dag, I.D., Sahin, A.S. and Korkmaz, A.K. (2010) Numerical Investigation of the Solution of Fisher's Equation via the B-Spline Galerkin Method. Numerical Methods for Partial Differential Equations, 26, 1483-1503.

[20] Rahman, K.R., Helil, N.H. and Yimin, A.R. (2010) Some New Semi-Implicit Finite Difference Schemes for Numerical Solution of Burgers Equation. International Conference on Computer Application and System Modeling, 14, 451-455.

[21] Gazdag, J.G. and Canosa, J.C. (1974) Numerical Solutions of Fisher's Equation. Journal of Applied Probability, 11, 445-457. https://doi.org/10.1017/S0021900200096236 
[22] Abdullaev, U.G. (1994) Stability of Symmetric Travelling Waves in the Cauchy Problem for the KPP Equation. Differential Equations, 30, 377-386.

[23] Logan, D.J. (1994) An Introduction to Nonlinear Partial Differential Equations. Wiley, New York.

[24] Evans, D.J. and Sahimi, M.S. (1989) The Alternating Group Explicit (AGE) Iterative Method to Solve Parabolic and Hyperbolic Partial Differential Equations. Annals of Numerical Fluid Mechanics and Heat Transfer, 2, 283-389.

[25] Tang, S.T. and Weber, R.O. (1991) Numerical Study of Fisher's Equations by a Petrov-Galerkin Finite Element Method. Journal of the Australian Mathematical Society Series B, 33, 27-38. https://doi.org/10.1017/S0334270000008602

[26] Khaled, K.A. (2001) Numerical Study of Fisher's Diffusion Reaction Equation by the Sinc Collocation Method. Journal of Computational and Applied Mathematics, 137, 245-255.

[27] Ames, W.F. (1965) Nonlinear Partial Differential Equations in Engineering. Academic Press, New York.

[28] Ames, W.F. (1969) Finite Difference Methods for Partial Differential Equations. Academic Press, New York.

[29] Noye, J.N. (1981) Nonlinear Partial Differential Equations in Engineering. NorthHolland Publishing Comp. Conference in Queen's College, University of Melbourne.

[30] Mittal, R.C. and Kumar, S.K. (2009) Numerical Study of Fisher's Equation by Wavelet Galerkin Method. International Journal of Computer Mathematics, 83, 287-298. https://doi.org/10.1080/00207160600717758

[31] Mehdi, M.B. and Khojasteh, D.S. (2013) A Highly Accurate Method to Solve Fisher's Equation. Pramana, 78, 335-346.

[32] Ablowitz, M.J. and Zeppetella, A.Z. (1979) Explicit Solutions of Fisher's Equation for a Special Wave Speed. Bulletin of Mathematical Biology, 41, 835-840. https://doi.org/10.1007/BF02462380

[33] Wasow, W.W. (1955) Discrete Approximation to Elliptic Differential Equations. Mathematical Physics, 6, 81-97.

[34] Schiesser, W.E. and Griffiths, G.W. (2009) A Compendium of Partial Differential Equation Models. Cambridge University Press, Cambridge. https://doi.org/10.1017/CBO9780511576270

[35] Whitham, G.B. (1974) Linear and Nonlinear Waves. John Wiley Sons, Hoboken.

[36] Babuska, I.B. (1968) Numerical Stability in Mathematical Analysis. IFIP Consress, North-Holland, Amsterdam, 11-23.

[37] Deghan, M.D., Asgar, H.A. and Mohammad, S.M. (2007) The Solution of Coupled Burgers Equations Using Adomian-Pade Technique. Applied Mathematics Computation, 189, 1034-1048.

[38] Lax, P.D. and Wendroff, B.W. (1960) Systems of Conservation Laws. Communications on Pure and Applied Mathematics, 13, 217-237. https://doi.org/10.1002/cpa.3160130205

[39] Kanti, P.K. and Lajja, V.L. (2011) A Note on Crank-Nicolson Scheme for Burgers Equation. Applied Mathematics, 2, 888-899.

[40] Roache, P.J. (1972) Computational Fluid Dynamics. Hermosa, Albuquerque.

[41] Mazumder, S.M. (2015) Numerical Methods for Partial Differential Equations: Fi- 
nite Difference and Finite Volume Methods. Academic Press, New York.

[42] Srivastava, V.K. and Tamsir, M.T. (2012) Crank Nicolson Semi Implicit Approach for Numerical Solution of Two Dimensional Coupled Non-Linear Burger's Equations. International Journal of Applied Mechanics and Engineering, 17, 571-581.

Submit or recommend next manuscript to SCIRP and we will provide best service for you:

Accepting pre-submission inquiries through Email, Facebook, LinkedIn, Twitter, etc. A wide selection of journals (inclusive of 9 subjects, more than 200 journals)

Providing 24-hour high-quality service

User-friendly online submission system

Fair and swift peer-review system

Efficient typesetting and proofreading procedure

Display of the result of downloads and visits, as well as the number of cited articles Maximum dissemination of your research work

Submit your manuscript at: http://papersubmission.scirp.org/

Or contact jamp@scirp.org 